ARTICLE

https://doi.org/10.1038/s41467-019-13149-7

\title{
A morphogenetic EphB/EphrinB code controls hepatopancreatic duct formation
}

M. Ilcim Thestrup ${ }^{1}$, Sara Caviglia (1) 1, Jordi Cayuso (1) 2, Ronja L.S. Heyne', Racha Ahmad', Wolfgang Hofmeister (103,4, Letizia Satriano ${ }^{5}$, David G. Wilkinson (1) ${ }^{2}$, Jesper B. Andersen (1) ${ }^{5} \&$ Elke A. Ober (iD ${ }^{1 \star}$

The hepatopancreatic ductal (HPD) system connects the intrahepatic and intrapancreatic ducts to the intestine and ensures the afferent transport of the bile and pancreatic enzymes. Yet the molecular and cellular mechanisms controlling their differentiation and morphogenesis into a functional ductal system are poorly understood. Here, we characterize HPD system morphogenesis by high-resolution microscopy in zebrafish. The HPD system differentiates from a rod of unpolarized cells into mature ducts by de novo lumen formation in a dynamic multi-step process. The remodeling step from multiple nascent lumina into a single lumen requires active cell intercalation and myosin contractility. We identify key functions for EphB/EphrinB signaling in this dynamic remodeling step. Two EphrinB ligands, EphrinB1 and EphrinB2a, and two EphB receptors, EphB3b and EphB4a, control HPD morphogenesis by remodeling individual ductal compartments, and thereby coordinate the morphogenesis of this multi-compartment ductal system.

\footnotetext{
${ }^{1}$ University of Copenhagen, NNF Center for Stem Cell Biology (DanStem), Blegdamsvej 3B, 2200 Copenhagen N, Denmark. ${ }^{2}$ The Francis Crick Institute, Neural Development Laboratory, 1 Midland Road, London NW1 1AT, UK. ${ }^{3}$ Odense University Hospital, Laboratory of Molecular and Cellular Cardiology, Department of Clinical Biochemistry and Pharmacology, Sdr. Boulevard 29, 5000 Odense C, Denmark. ${ }^{4}$ University of Southern Denmark, Department of Cardiovascular and Renal Research, Winsloewparken 21, 5000 Odense C, Denmark. ${ }^{5}$ University of Copenhagen, Biotech Research and Innovation Centre, Copenhagen, Denmark. *email: elke.ober@sund.ku.dk
} 
critical step in establishing a functional digestive system is the connection of the accessory organs to the intestine. During development, the hepatopancreatic ductal (HPD) system connects the liver, gallbladder (GB) and pancreas to the digestive tract. The HPD system is essential for food processing and uptake of nutrients, as it transports the bile produced in the liver, as well as pancreatic enzymes and electrolytes to the intestine, but little is known about how the HPD develops. It is a multi-component system consisting of the extrahepatic duct (EHD), cystic duct (CD), common bile duct (CBD) and extrapancreatic duct (EPD; Fig. 1f) ${ }^{1}$.
A subgroup including $\mathrm{EHD}, \mathrm{CD}$ and $\mathrm{CBD}$, as well as the gallbladder $\mathrm{GB}$ are known as the extrahepatic biliary ducts (EHB). The CBD coming from the liver and the EPD from the pancreas converge into the hepatopancreatic ampulla (HPA), also known as Ampulla of Vater in humans, connecting the HPD system to the intestine. This organization and morphology of the HPD system is conserved between fish and amniotes, however, in mammals the EHDs are commonly referred to as right or left ducts and connecting common duct, and the EPD as pancreatic duct ${ }^{1,2}$. Congenital or environmentally caused malformation of the HPD, such as ductal atresia
$46 \mathrm{hpf}$

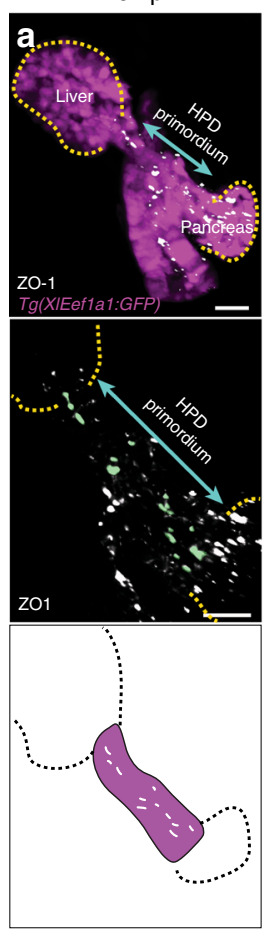

g

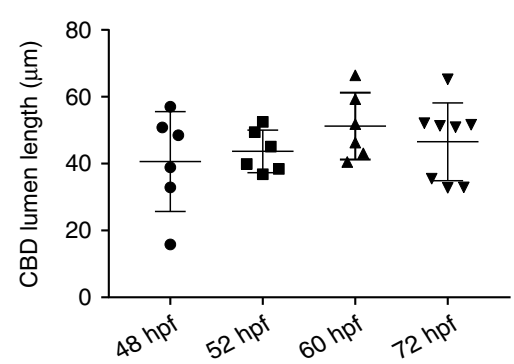

$52 \mathrm{hpf}$

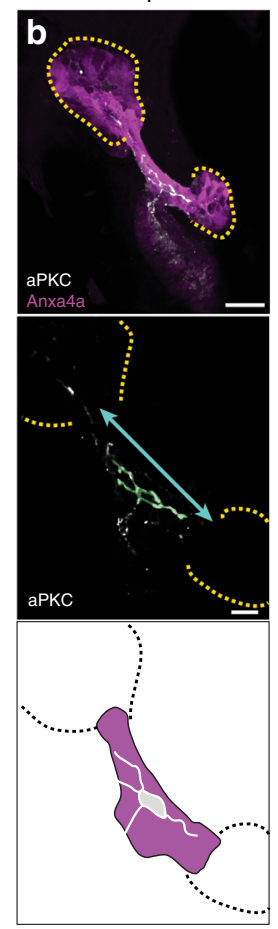

60 hpf

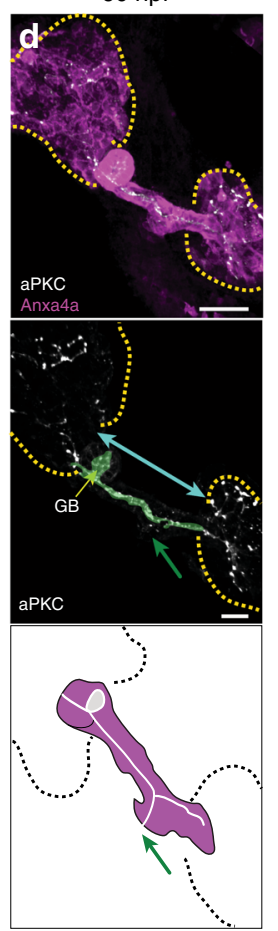

72 hpf

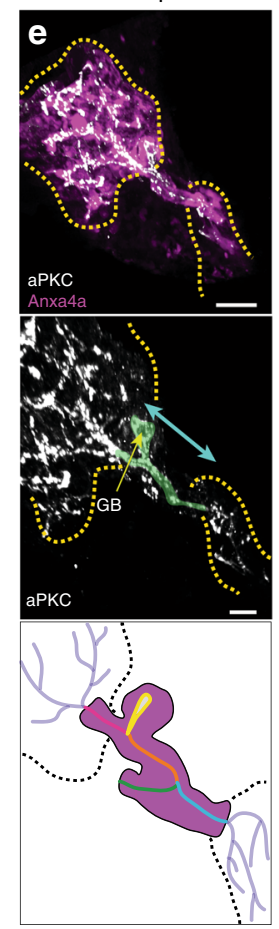

$5 \mathrm{dpf}$

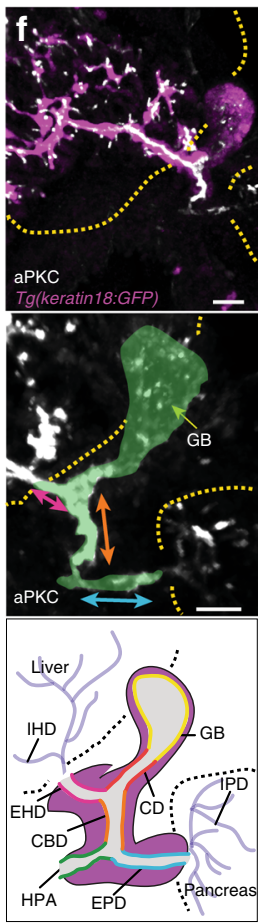

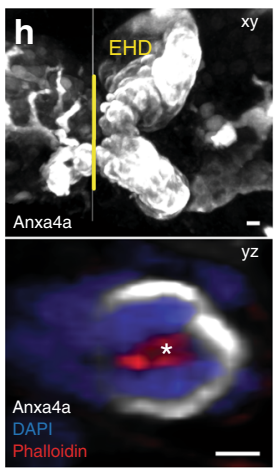
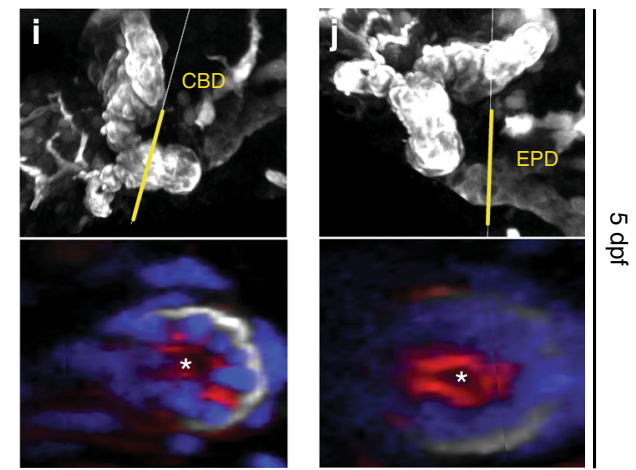

Fig. 1 The HPD system forms by de novo tubulogenesis. a The forming HPD is visualized by pan-endodermal Tg(XIa.Eef1a1:GFP) (magenta) and ZO-1 (gray) labels the first junctional aggregates in the HPD primordium (blue arrow) at $46 \mathrm{hpf}$. b Ductal endoderm expression of Anxa4 (magenta) and apical aPKC (gray) visualize HPD morphology and nascent microlumina within the prospective CBD and EPD $(n=17, N=3)$. c The HPD system elongates and the gallbladder anlage (yellow arrow) becomes detectable at $52 \mathrm{hpf}$. The immature EHB and HPA lumina show gaps and luminal loops compared to the EPD exhibiting a continuous immature lumen $(n=21, N=3)$. The HPA connects at two points (green arrows). d At 60 hpf, all ductal compartments and a sphere-like gallbladder display a continuous emerging lumen, including a single connection of the HPA (green arrow), but excluding the EHD ( $n=29, N=$

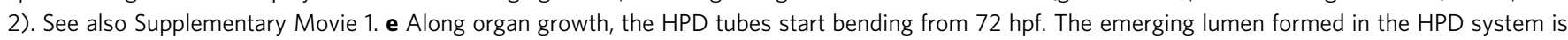
continuous and connected to both IHD and IPD luminal network $(n=11, N=2)$ f transgenic keratin18:GFP expression visualizes the compact EHB and IHD at $5 \mathrm{dpf}(n=13, N=4)$, see also Supplementary Movie 8. Schematic overviews of HPD differentiation (a-f): starting with junctional aggregates and resulting in a continuous lumen spanning the HPD system. From 52 hpf individual HPD compartments are distinguishable: extrahepatic duct (EHD, pink); cystic duct (CD, red); gallbladder (GB, yellow); common bile duct (CBD, orange); extrapancreatic duct (EPD, blue); hepatopancreatic ampula (HPA, green). Scale bars: $\mathbf{a}-\mathbf{f}=10 \mu \mathrm{m} . \mathbf{g}$ Lumen length quantification of the differentiating CBD between 48 and 72 hpf. h-j At 5 dpf, cortical actin indicates open lumina in the wild-type EHD, CBD and EPD; scale bars $=10 \mu \mathrm{m}$. Magnified cross-sections show open lumina (asterisk) of indicated ductal domains (yellow lines); scale bars $=5 \mu \mathrm{m}$. Error bars show SEM; $n=$ sample number, $N=$ number of experiments. Source data are provided as a Source Data file 
caused by duct obstruction or paucity compromise organ function and cause chronic inflammation ${ }^{3-6}$. Pancreatobiliary junction malformations can result in a two-way reflux of bile and pancreatic juice, leading to inflammation and subsequently malignant transformation ${ }^{7}$. This underscores the importance of establishing the ductal compartments and their junctions, however, the causes of congenital HPD malformations are largely unknown.

HPD progenitors arise from an endodermal cell population located between the liver and pancreas anlagen. In mouse, this population co-expresses transcription factors Sox 17 and Pdx1, with Sox17 being essential for EHB duct formation ${ }^{8}$. The latter acts in concert with other transcription factors, including Hes1, Hnf6, Hnf1 $\beta$ and Hhex, whose deletion causes HPD malformations ranging from CBD enlargement and GB agenesis to replacement with pancreatic or duodenal cells ${ }^{9-14}$. HPD progenitors in zebrafish are similarly located between the liver and pancreas and express transcription factors Pdx1, Prox1, and Sox9 $b^{14,15}$. Intriguingly, lack of Sox9b leads to HPD malformation and ectopically differentiating hepatic and pancreatic cells in the HPD ${ }^{15,16}$. Zebrafish mutants for mesodermal $f g f 10$ exhibit similar cellular plasticity in the HPD, indicating that mesoderm-endoderm interactions are essential for HPD differentiation ${ }^{2,10}$. The morphological emergence of the HPD coincides with the downregulation of Proxl in HPD progenitors and membrane localisation of calcium binding protein AnnexinA4 (Anxa4) ${ }^{2,17}$. Although the transcriptional network controlling HPD development is emerging ${ }^{1,18}$, the morphogenetic events underlying duct differentiation and their molecular regulators are largely unknown. Two classic hypotheses describe the formation of the HPD either by budding and invagination of the foregut epithelium, or by differentiation from a solid tissue (e.g. cord) by subsequent vacuolization and lumen formation. Histological analysis of human embryonic tissue favors that a bile duct lumen buds from the foregut, without transitioning through a solid tissue stage ${ }^{19}$. Due to limited sample accessibility and developmental stages, current understanding is incomplete and an in-depth analysis is necessary.

Eph receptor tyrosine kinases and their cognate Ephrin ligands are regulators of diverse cellular functions, such as cell adhesion, migration and proliferation, which are critical for organ morphogenesis and homeostasis ${ }^{20-22}$. EphB receptors interact primarily with B-type transmembrane Ephrin ligands ${ }^{21}$. Their interaction can uniquely trigger bidirectional signaling upon cell contact, with EphB-expressing cells activating forward signaling and EphrinBbearing cells eliciting reverse signaling. Little is known about their function in duct formation, whereas key roles in epithelial tissues encompass localization of tight and adherens junctions proteins in the Xenopus epithelial ectoderm ${ }^{23,24}$, cell sorting and positioning by local actomyosin contractility at the Xenopus notochord-presomitic mesoderm interface ${ }^{25}$, integrin clustering and extracellular matrix assembly in zebrafish somite boundary morphogenesis ${ }^{26}$. In the context of inter-rhombomeric boundaries Ephs and Ephrins inhibit cell intermingling and maintain boundary sharpness by promoting actomyosin cable formation ${ }^{27,28}$. Co-expression of multiple Ephs and Ephrins can confer signaling strength to activate cell detachment during repulsion ${ }^{29}$. We previously showed that EphrinB1 and EphB3b control directional liver progenitor migration into the liver bud by a repulsion-based mechanism during early liver development in zebrafish ${ }^{30}$. Embryos with impaired EphrinB1 or EphB3b function exhibit dysmorphic HPD systems at later stages, suggesting a hitherto unknown function for $\mathrm{EphB} / \mathrm{EphrinB}$ signaling in $\mathrm{HPD}$ morphogenesis.

Here, we present a high-resolution analysis of HPD tube morphogenesis in zebrafish. We show that a single lumen arises by de novo lumen formation from a solid cord-like primordium of unpolarized cells by a cord hollowing mechanism. This multistep process is driven by dynamic cell rearrangements, which include cell intercalation, promoted by non-muscle myosin II activity. Using genetic approaches, we provide evidence that these processes are regulated by a morphogenetic EphB/EphrinB code of multiple EphrinB ligands and EphB receptors controlling duct differentiation and GB formation in a region-specific fashion.

\section{Results}

The HPD system forms by de novo lumen formation. To elucidate the morphogenetic process of HPD formation we first investigated whether the ducts arise from an existing epithelial sheet by a budding or wrapping mechanism, or from unpolarized cells by de novo lumen formation ${ }^{31}$. Overall HPD morphology was visualized by transgenic expression of pan-endodermal XlaEef1a1:GFPs854, ductal keratin18:GFP (specific for hepatic domains) or immunolabeling of Anxa4, recognized by the 2F11 antibody ${ }^{17}$. Cell polarity and lumen formation were assessed by staining for atypical protein kinase $\mathrm{C} \lambda(\mathrm{aPKC})$ a member of the PAR protein/aPKC complex that localizes to the apical membrane of epithelial cells, or for the tight junction protein ZO-1. Zebrafish larvae were analyzed between 46 hours post fertilization (hpf), when the HPD primordium emerges, and 5 days post fertilization (dpf), when larvae start to feed (Fig. 1a-f). All HPD compartments were assigned based on morphological landmarks. The first signs of tube formation are $\mathrm{ZO}-1$ aggregates, which appear randomly distributed along the central axis of the emerging HPD primordium at $46 \mathrm{hpf}$ (Fig. 1a), indicating that HPD progenitors start to polarize. As early as $48 \mathrm{hpf}$, the CBD and EPD are distinguishable as the first domains of the HPD, at the proximal end of the liver and pancreas, respectively (Fig. 1b). Multiple, short and disconnected aPKC-positive apical structures within both domains indicate the formation of immature luminal pockets, reminiscent of microlumina described in the murine pancreas $^{32,33}$. In parallel, the HPD-to-gut connection is established through nascent HPA apical pockets at multiple contact points. Hence, all HPD ducts form de novo and not by a budding process from the existing foregut epithelium (Fig. 1a, b). At 52 hpf, the GB primordium is detectable by dense group of aPKC foci and its round morphology at the distal end of the CBD (Fig. 1c), which later will connect to the EHD-CBD lumen via the $\mathrm{CD}$. At this stage, aPKC-positive apical pockets in the CBD and the EPD form a semi-continuous nascent lumen, including gaps and loops of parallel apical structures. The HPD is in most cases connected to the gut at two points revealing that the HPA is still immature (Fig. 1c). Between 52 and $60 \mathrm{hpf}$, duct maturation results in the consolidation of a single continuous lumen connecting all compartments of the HPD, and duct lengthening (Fig. 1d, g, Supplementary Movie 1). Finally, a refined EHD lumen connecting the HPD to the intrahepatic ductal (IHD) network is clearly distinguishable by $72 \mathrm{hpf}$ (Fig. 1e). Subsequently, the $\mathrm{CBD}$ and $\mathrm{EPD}$ bend as the connected organs grow and their position within the body adjusts (Fig. 1e-g). Lumen inflation of the HPD compartments occurs after $72 \mathrm{hpf}$ and by 5 dpf (Fig. 1h-j, Supplementary Movie 8), likely due to apical secretion of non-bile fluids from the ductal epithelial cells and bile secretion and transport from the liver starting at $5 \mathrm{dpf}^{34}$. Notably, aPKC staining before $5 \mathrm{dpf}$ indicates HPD cell polarization and lumen formation, however not necessarily an open patent lumen.

These findings show that HPD morphogenesis encompasses a multi-step process, starting with scattered junctional aggregates, which transition to form apical pockets and then fuse into immature ductule morphology, including complex loop-shaped apical structures, and finally remodel into a single central lumen (Fig. 8c). This suggests that HPD tubes form from a solid cordlike primordium, which undergoes de novo lumen formation following a cord hollowing process. 
EphrinB1 and EphB3b control HPD morphogenesis. To elucidate the molecular mechanisms controlling HPD morphogenesis, we focused on EphrinB1 and EphB3b as candidates, because embryos with compromised function of either factor exhibit dysmorphic HPD systems ${ }^{30}$ and $\mathrm{EphB} /$ EphrinB signaling governs complex morphogenetic processes ${ }^{21}$. For the analyses, we used ephrinb1 $1^{\text {nim } 26}$ mutants and generated an ephb3b $b^{\text {nim } 27}$ genetic mutant by Crispr/Cas9 genome editing (Supplementary Fig. 1). We analyzed duct morphogenesis in ephrinb1 and ephb3b mutant embryos at key stages of HPD formation by immunostaining for Proxl, which labels hepatic and pancreatic progenitors, HNF4a for differentiating hepatocytes, and aPKC visualizing apical membranes (Fig. 2, Supplementary Fig. 2). Similar to controls, aPKC-positive apical pockets emerge in ephrinb1 and ephb3b
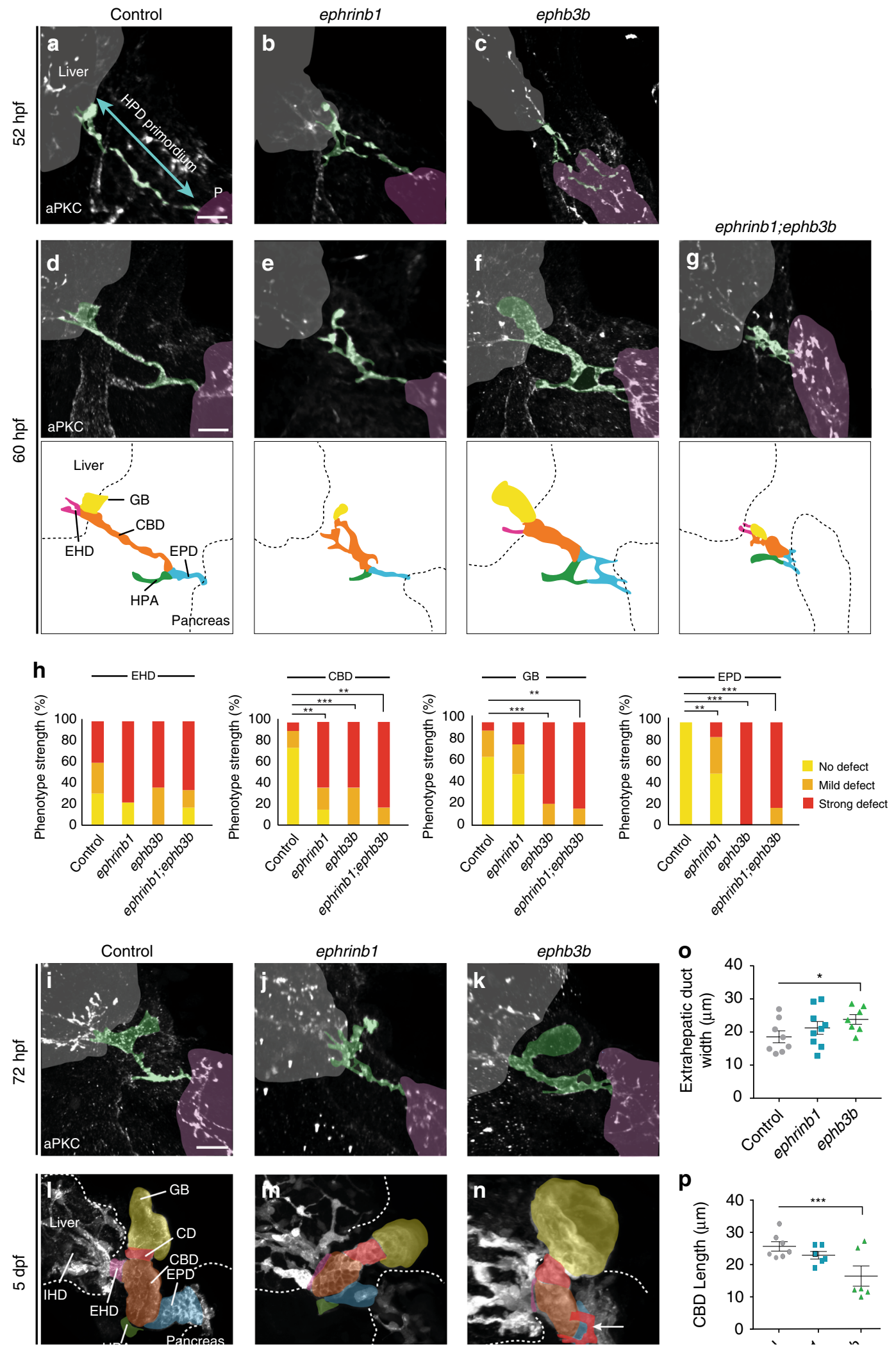
Fig. 2 EphrinB1 and EphB3b control HPD remodeling in a spatiotemporal fashion. Compared to sibling controls at 52 hpf (a), emerging ducts are less mature in ephrinb1 mutants with more disconnected luminal pockets in EHB and HPA, visualized by apical aPKC (white, $n=16, N=3)$ (b). c HPD luminal pockets are similarly disrupted in ephb3b mutants at $52 \mathrm{hpf}$, including the EPD $(n=17, N=2)$. d Disconnected apical structures resolve into a single immature lumen by $60 \mathrm{hpf}$ in controls $(n=29, N=2)$. e Disrupted remodeling causes enlarged aPKC structures and luminal loops in all EHB domains in ephrinb1 mutants, while the EPD appears normal $(n=20, N=2)$. $\mathbf{f}$ Resolution of apical structures in ephb3b mutants is compromised in all HPD domains, including the EPD $(n=12, N=1)$. $\mathbf{g}$ All HPD domains exhibit defective tube remodeling in ephrinb1;ephb3b mutants $(n=7)$. Schematics $(\mathbf{d}-\mathbf{f})$ show HPD tube phenotypes at $60 \mathrm{hpf}$, see below for domain color-code. $\mathbf{h}$ Quantification of domain-specific HPD tube formation defects in controls ( $n=12$ ), ephrinb1 $(n=14)$, ephb3b $(n=14)$ and ephrinb1;ephb3b $(n=7)$ mutants at 60 hpf. i-n Early domain-specific defects persist in ephrinb1 and ephb3b mutants at 72 hpf (i-k; controls: $n=11, N=2$; ephrinb1: $n=5$; ephb3b: $n=12, N=1$ ) and $5 \mathrm{dpf}(\mathbf{I}-\mathbf{n}$; control: $n=10, N=2$; ephrinb1: $n=6, N=1 ;$ ephb3b: $n=10, N=1)$. 30\% ephb3b mutants show in addition ectopic ducts (red; white arrow) bifurcating from the main HPD. Quantification of EHD width (o) and CBD length (p) in ephrinb1 and ephb3b mutants at $5 \mathrm{dpf}$. I-n Anxa4 (gray) visualizes the HPD system at $5 \mathrm{dpf}$. HPD domains are color-coded based on morphological landmarks (e.g., HPA): $\mathrm{EHD}=$ pink; $\mathrm{CD}=$ red; gallbladder = yellow; $\mathrm{CBD}=$ orange; $\mathrm{EPD}=$ blue; $\mathrm{HPA}=$ green. Scale bar $=20 \mu \mathrm{m} ; n=$ sample number, $N=$ number of experiments; Statistical test: $\mathbf{h}=$ Fisher Exact, $\mathbf{0}, \mathbf{p}=$ Student's $t$-test. Errors bar show $\mathrm{SEM} ;{ }^{*} p<0,05,{ }^{\star \star \star} p<0,001$. Liver and pancreas stainings for a-f and i-k are shown in Supplementary Fig. 2. Supplementary movies 1-4 and 8-10 show HPD phenotypes at 60 hpf and 5 dpf. Source data are provided as a Source Data file

Table 1 Domain-specific classification of HPD tube formation defects

\begin{tabular}{lll} 
HPD domain & $\begin{array}{l}\text { Score 1 = mild defect } \\
\text { (ductal domains show only one of below defects) }\end{array}$ & $\begin{array}{l}\text { Score 2 severe defect (ductal domains show } \\
\text { more than one of below defects) }\end{array}$ \\
\hline Extrahepatic duct (EHD) & Two branches, thinner, enlarged or gap & More than two branches, loops \\
Gallbladder (GB) & $\begin{array}{l}\text { A bit longer/more inflated, gap, more than one branch attached } \\
\text { distally or enlarged cystic duct }\end{array}$ & Much longerinflated \\
Common bile duct (CBD) & Long spike, loop, gap or more inflated & Cystic, loops \\
Extrapancreatic duct (EPD) & Long spike, loop, gap or more inflated & Cystic, loops
\end{tabular}

mutants at $48 \mathrm{hpf}$, suggesting that HPD progenitors form a HPD primordium, polarize and initiate the formation of nascent tubes. However, these structures appear slightly disorganized (Supplementary Fig. 2a-c). By $52 \mathrm{hpf}$, the aPKC structures in the prospective CBD and CBD-EPD-HPA junction are often associated with more disruptions or loops in most ephrinb1 mutants compared to wild-type (Fig. 2a, b). In contrast to wild-type and ephrinb1 mutants, ephb3b mutants show aPKC loops also in the EPD (Fig. $2 \mathrm{a}-\mathrm{c}$ ). The resolution of these immature lumina into a single continuous lumen primordium in all HPD regions occurs in wild-type by $60 \mathrm{hpf}$, however, is severely compromised in the absence of EphrinB1 and EphB3b (Fig. 2d-f, Supplementary Movie 1-3). Phenotype variability and penetrance were assessed by quantification of defect severity in the individual HPD domains (Fig. 2h, Table 1, Supplementary Fig. 3). Notably, a single nascent EPD lumen is resolved in most ephrinb1 mutants (Fig. 2d-e), whereas this is still disorganized and web-like in ephb3b embryos (Fig. 2f). ephrinb1;ephb3b double mutants exhibit defects in all HPD domains similar to ephb3b (Fig. 2g, h, Supplementary Movie 4), disregarding additive or synergistic interactions and corroborating that $\mathrm{EphB} 3 \mathrm{~b}$ regulates morphogenesis of all HPD domains, while EphrinB1 mediates this process only in a subset of domains. Unresolved loops, enlarged aPKC/luminal structures, an amorph EHD or combinations of these defects, are in both mutants frequently not remodeled by 72 hpf (Fig. 2i-k). Next, we asked whether the observed HPD defects in ephrinb1 and ephb3b mutants persist when digestive activity is starting at around $5 \mathrm{dpf}$. Anxa4 immunostainings showed dysmorphic ductal compartments in both ephrinb1 and ephb3b mutants, including significantly altered EHD width and CBD length, corresponding with earlier regional remodeling defects (Fig. 2l-p, Supplementary Movies 8-10). In addition, ephb3b mutants display larger GB and occasionally ectopic small ducts branching from the EHD, CBD or EPD (30\%, Fig. 2n).

Altogether these findings show that EphrinB1 and EphB3b control HPD morphogenesis in a domain-specific manner:
EphrinB1 governs EHB tube morphogenesis, whereas EphB3b is required for the formation of the entire $\mathrm{HPD}$, including the EPD. These only partially overlapping functions imply that HPD morphogenesis may require additional EphrinB ligands and EphB receptors.

EphrinB1 and EphB3b regulate dynamic CBD cell rearrangements. Our analysis of lumen formation suggests that the HPD system arises by de novo tube formation. To identify the underlying cell behaviors and morphogenetic mechanism, we first examined the organization of the HPD, focusing on the CBD, by co-staining for apical aPKC and pan-Cadherin, which localizes to lateral membranes in epithelia and is expressed in the HPD endoderm ${ }^{2}$. At $48 \mathrm{hpf}$, the HPD represents a solid cord consisting of 3-4 cell layers (Fig. 3a), which transforms into a single-layered epithelial duct by $60 \mathrm{hpf}$ (Fig. 3b). Concomitantly, CBD length increases significantly between 52 and $60 \mathrm{hpf}$ in controls, while in both ephrinb1 and ephb3b mutants this extension fails to occur during this time window (Fig. 3e). To distinguish whether CBD tube formation is driven by active cell rearrangement and intercalation, or other mechanisms, such as apoptosis characteristic for cavitation, we analyzed epithelial morphology.

At $60 \mathrm{hpf}$, when a continuous immature lumen extends throughout the ductal system, epithelial cells are aligned in a single layer with their apical domains forming the arising central lumen (Fig. 3b). Cell shape analyses of individual cells with luminal contribution in wild-type revealed a subset of teardropshaped cells during duct remodeling stages (Supplementary Fig. 4a). A teardrop-like morphology is a hallmark of radially intercalating cells ${ }^{35}$, suggesting that those cells are actively rearranging within the epithelium. In both ephrinb1 and ephb3b mutants, epithelial organization is disrupted, marked by altered irregular cell shapes and a multi-layered cell organization in areas of immature apical loops (Fig. 3c, d). Ductal cells contributing to ductal loops appear to have a single largely extended or multiple apical domains (Fig. 3c). Cell shape analysis of the CBD revealed 

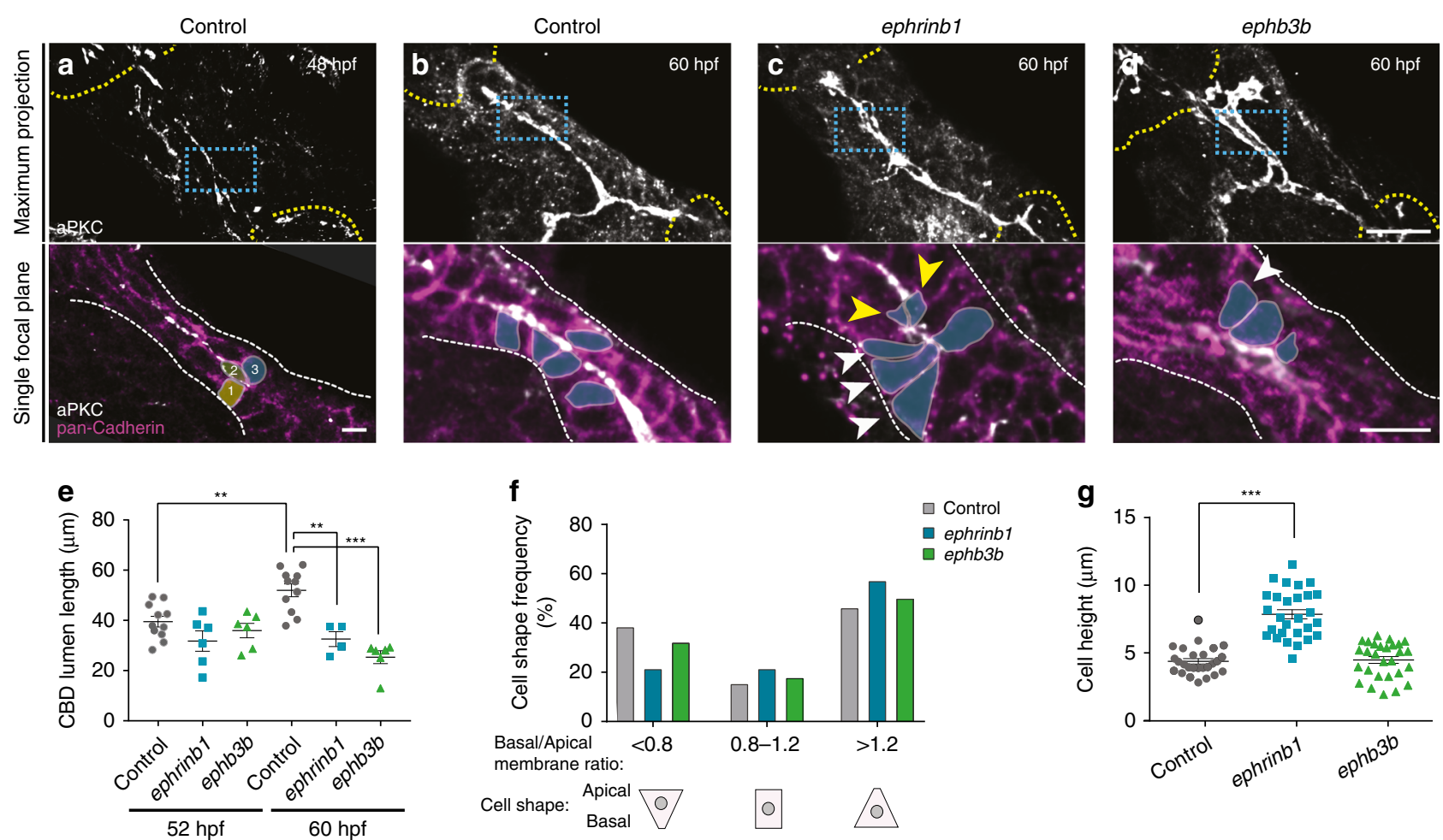

Fig. 3 Ductal cell rearrangement and lumen resolution require EphrinB1 and EphB3b. a-d Overview of the forming HPD epithelium by maximum projection of confocal stacks stained for PanCadherin (magenta) aPKC (white) in control and ephrinb1 and ephb3b mutants. Blue dashed squares indicate magnified CBD containing areas. a At 48 hpf, PanCadherin shows multiple cell layers in CBD (e.g., colored cells 1-3). b By 60 hpf, the CBD is largely a single-layered epithelium with apical domains facing the central lumen. c, d ephrinb1 and ephb3b CBDs show disrupted epithelial organization, including luminal loops, with cells with multiple or extended apical domains (yellow arrowheads), and teardrop shaped-cells with reduced apical domains (white arrowhead). e Quantification of the longest continuous aPKC staining as a measure for CBD lumen elongation in controls, ephrinb1 and ephb3b mutants between 52 and $60 \mathrm{hpf}$. $\mathrm{Basal} /$ apical membrane ratio was used to quantify CBD cell shapes in control $(n=26$ cells), ephrinb1 ( $n=28)$ and ephb3b ( $n=28)$ embryos at 60 hpf. $\mathbf{g}$ Quantification of CBD cell height (distance between apical and basal membranes) in control $(n=26$ cells), ephrinb1 ( $n=28)$ and ephb3b ( $n=28$ ) embryos at 60 hpf. Scale bars: $\mathbf{a}=5 \mu \mathrm{m}, \mathbf{b}$-d $=20 \mu \mathrm{m}$. Statistical test: $\mathbf{e}, \mathbf{g}=$ Student's $t$-test, $\mathbf{f}=$ Fisher Exact. Errors bar show SEM; ${ }^{\star \star} p<0,01,{ }^{\star \star \star} p<$ 0,001. Source data are provided as a Source Data file

a clear increase of teardrop-shaped cells facing the apical lumen in both ephrinb1 and ephb3b mutants at the expense of those with opposite orientation and more columnar cells (Fig. 3f). In both mutants, these changes are associated with an increase in cell height (Fig. 3g). Impaired cell rearrangement in ephrinb1 and $e p h b 3 b$ mutants results in a failure of CBD extension (Fig. 3e). Finally, active cell rearrangement is supported by sparse labeling of small clones with the membrane-tethered fluorescent protein lyn-Tomato in wild-type, revealing HPD cells extending small filopodia-like protrusions, including several towards the forming lumen (Supplementary Fig. 4b). Given the known role of EphB/ EphrinB signaling in cell proliferation ${ }^{21}$, we assessed its contribution to HPD morphogenesis. Neither cell proliferation rate nor overall HPD cell number are significantly changed in ephrinb1 and ephb3b mutants compared to controls (Supplementary Fig. 6), indicating that EphrinB1 and EphB3b control HPD morphogenesis mainly by alternative mechanisms. Together these data indicate that scattered apical pockets remodel and coalesce into a continuous nascent lumen by dynamic cell shape changes and active intercalation-like cell rearrangement, transforming a compact cord-like HPD primordium into a mono-layered epithelial duct. EphrinB1 and EphB3b play a crucial role in regulating these cell shape changes essential for HPD tubulogenesis.

Non-muscle myosin activity mediates HPD lumen remodeling. The dynamic changes in epithelial organization of the HPD during duct differentiation and the related defects in ephrinb1 and $e p h b 3 b$ mutants suggest an active contribution of cytoskeletal remodeling and actomyosin contractility to the tube maturation process $^{36}$. First, we assessed whether myosin II contractility is required for HPD formation by incubating wild-type embryos with the selective pMLC inhibitor blebbistatin during lumen remodeling (Fig. 4a). Blebbistatin incubation consistently caused luminal gaps and loops in the HPD of $60 \mathrm{hpf}$ embryos (Fig. 4b-d), reminiscent of the lumen remodeling defects observed in ephrinb1 and ephb3b mutants. Moreover, remodeling defects are associated with rounded and/or elongated epithelial cells and a significantly elongated CBD (Fig. 4e). These findings show that myosin II contractility is required for HPD remodeling into a mature tube, possibly contributing to multiple steps of cell shape changes leading to the rearrangement of polarizing cells to form a central lumen. To elucidate the function of myosin activity, we determined its subcellular distribution. Staining showed that actin and active non-muscle myosin II, visualized by phosphorylation of its regulatory light chain (pMLC), are enriched apically and at lower levels at lateral and likely at basal membranes during stages of HPD remodeling (Fig. 4f). Myosin contractility is therefore a good candidate to regulate apical domain size in the differentiating HPD. We determined apical pMLC levels focusing on ephrinb1 mutants, which exhibit distinct cell shape change defects in the CBD at $60 \mathrm{hpf}$ (Fig. 3f, g). Quantification of staining intensity showed an up to six-fold increase of apical pMLC levels in ephrinb1 mutants compared to 

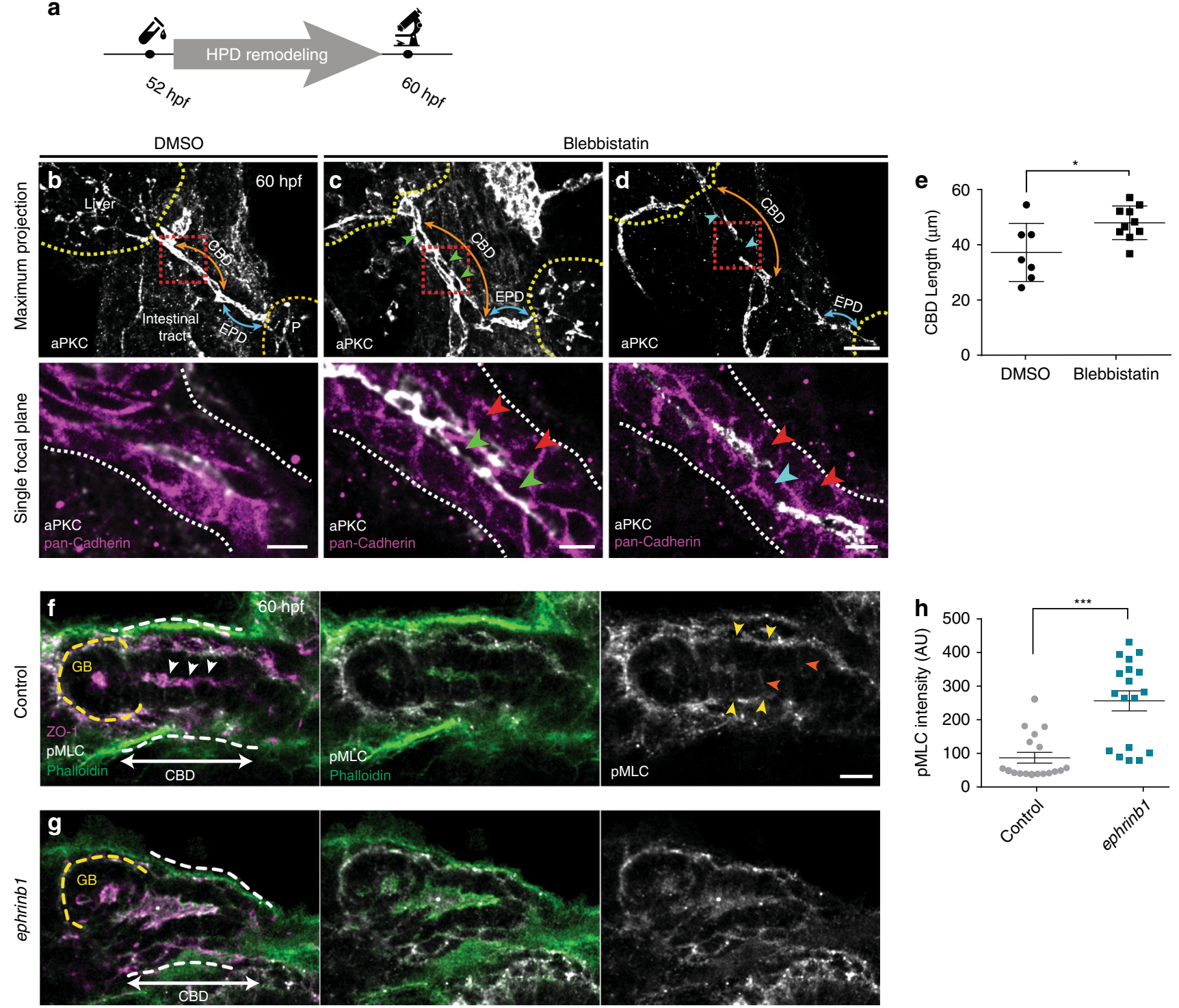

Fig. 4 HPD differentiation requires EphrinB1-controlled actomyosin contractility. a Experimental scheme for $65 \mu \mathrm{M}$ blebbistatin incubation during HPD remodeling from 52-60 hpf. b-d Blebbistatin-treated embryos show two types of EHB epithelial defects: $\mathbf{c}$ extensive luminal loops (45.5\%, $n=11, N=2)$ and $\mathbf{d}$ gaps in the emerging apical lumen (45.5\%, $n=11, N=2$; and $9 \%$ wild-type like). The nascent EPD lumen is mostly resolved, consistent with the treatment window; scale bars $=15 \mu \mathrm{m}$. PanCadherin staining (magenta) of magnified views of CBD (red dashed box) shows rounder epithelial cells (red arrowheads), a multi-layered configuration with loops and cells exhibiting extended apical domains (green arrowheads) or gaps and cells with multiple apical domains (blue arrowhead) in treated embryos compared to controls; scale bars $=5 \mu \mathrm{m}$. e Quantification of CBD length shows significant increase upon blebbistatin treatment compared to controls. $\mathbf{f}, \mathbf{g}$ Actin, pMLC and ZO-1 localize apically (white arrowheads) in the forming CBD; whether pMLC is at the apical membrane and/or the junctional belt cannot be distinguished. pMLC is at the duct mesoderm border (yellow arrowheads) and lower levels at the lateral membrane (orange arrowheads) 60 hpf; scale bars $=5 \mu \mathrm{m}$. h Quantification of apical pMLC expression in CBD cells of $60 \mathrm{hpf}$ control $(n=3)$ and ephrinb1 embryos $(n=3)$. For quantification strategy see Supplementary Fig. 5. Statistical test: $\mathbf{e}, \mathbf{h}=$ Student's $t$-test. Error bars show SEM; ${ }^{\star} p<0,05$,

${ }_{\star \star \star} p<0,001$. Source data are provided as a Source Data file

controls, suggesting hyper-contracted apical domains contribute to the altered cell shapes in the CBD (Fig. 4f-h, Supplementary Fig. 5). These data demonstrate that EphrinB1 contributes to HPD cell rearrangement and lumen resolution by directly or indirectly regulating apical pMLC levels and distribution.

EphB/EphrinB signaling is required for HPD lumen remodeling. To distinguish whether HPD malformations in ephrinb1 and ephb3b mutants are the result of a requirement for EphrinB1 and EphB3b in HPD remodeling or secondary to earlier progenitor positioning defects ${ }^{30}$, we blocked EphB/EphrinB signaling specifically during the time window of duct remodeling. For this we expressed EphrinB1 ${ }^{\mathrm{EC}}$, the extracellular domain of EphrinB1 which acts as a secreted, dominant-negative form ${ }^{37}$. For conditional ubiquitous expression transgenic UAS:ephrin$B 1^{E C}$ fish were crossed with hsp70l:Gal4 fish and the embryos exposed to heat-shock at key stages of HPD remodeling and assessed at $60 \mathrm{hpf}$ when a single HPD lumen is established (Fig. 5a, b). The majority of manipulated embryos display severe morphological HPD defects upon blocking EphB/EphrinB signaling around the onset of remodeling at $50 \mathrm{hpf}$ or when remodeling is ongoing at $54 \mathrm{hpf}$. Defects include luminal loops, dysmorphic and enlarged immature luminal domains 
a

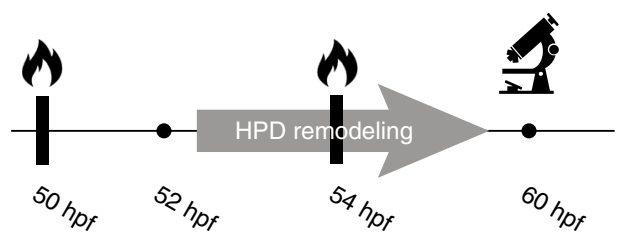

Control

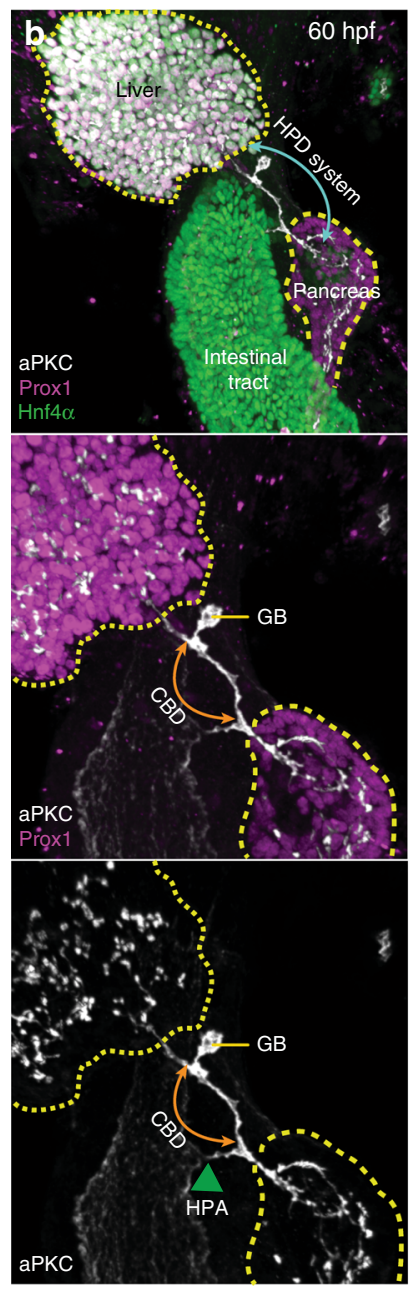

UAS:ephrinb1 $1^{E C}$
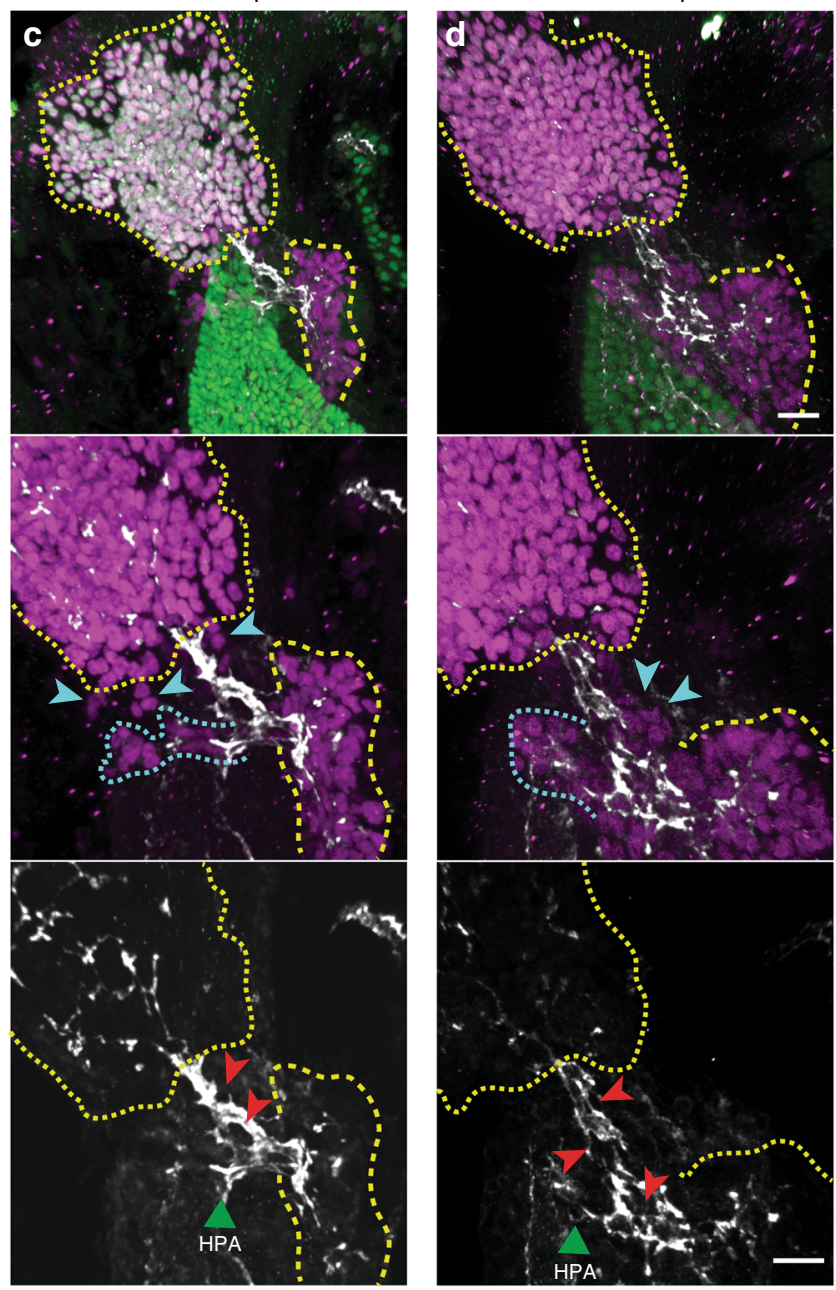

Fig. 5 EphB/EphrinB signaling controls HPD lumen morphogenesis independently of other morphogenetic roles. a Conditional ephrinb7EC expression is

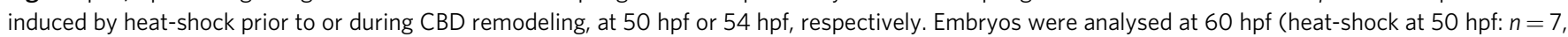
heat-shock at 54 hpf: $n=7$, both $N=1$ ). b-d Hnf $4 \alpha$ (green), Prox1 (magenta) and aPKC (gray) labeling show that unlike in controls (b), conditional ephrinb1EC expression at $50 \mathrm{hpf}$ (c) and $54 \mathrm{hpf}$ (d) leads to HPD, liver and pancreas morphology defects, ectopic Prox1-positive cells throughout the HPD

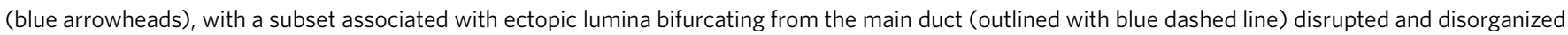
HPD lumen (red arrowheads, c, d). Yellow dotted lines: liver, yellow dashed line: pancreas; blue arrow: HPD system, orange arrow: CBD, green arrowhead: HPA

throughout the HPD and a severely hypoplastic or absent GB (Fig. 5c, d). In addition, in most embryos the morphology of the pancreas is dysmorphic, with some embryos displaying ectopic groups of Prox $1^{+} / \mathrm{Hnf} 4 \alpha^{-}$cells that frequently appear connected by aPKC ${ }^{+}$ductal structures to the EPD or pancreas (Fig. $5 c, d$ ), suggesting impaired integrity of the pancreatic epithelium and its expansion into ectopic locations. Hence, EphB/EphrinB signaling is required for HPD duct remodeling and EphrinB1 and EphB3b could play multiple independent and sequential roles in liver, pancreas and HPD development. Moreover, phenotypes upon blocking EphB/EphrinB signaling are often more severe than those observed in ephb3b and in particular ephrinb1 mutants alone, supporting that additional EphrinBs and EphBs may be involved in HPD development.

EphrinB2a and EphB4a regulate GB and CBD formation. In light of the only partial overlap of ephrinb1 and ephb3b mutant HPD phenotypes and the more severe malformations observed upon blocking EphB/EphrinB signaling, we assessed the involvement of additional EphrinB ligands and EphB receptors in HPD development. First, we determined the spatial expression of EphrinB1 and EphB3b by immunostaining. During the onset of HPD tube formation, at $48 \mathrm{hpf}$, EphrinB1 is expressed in the liver 

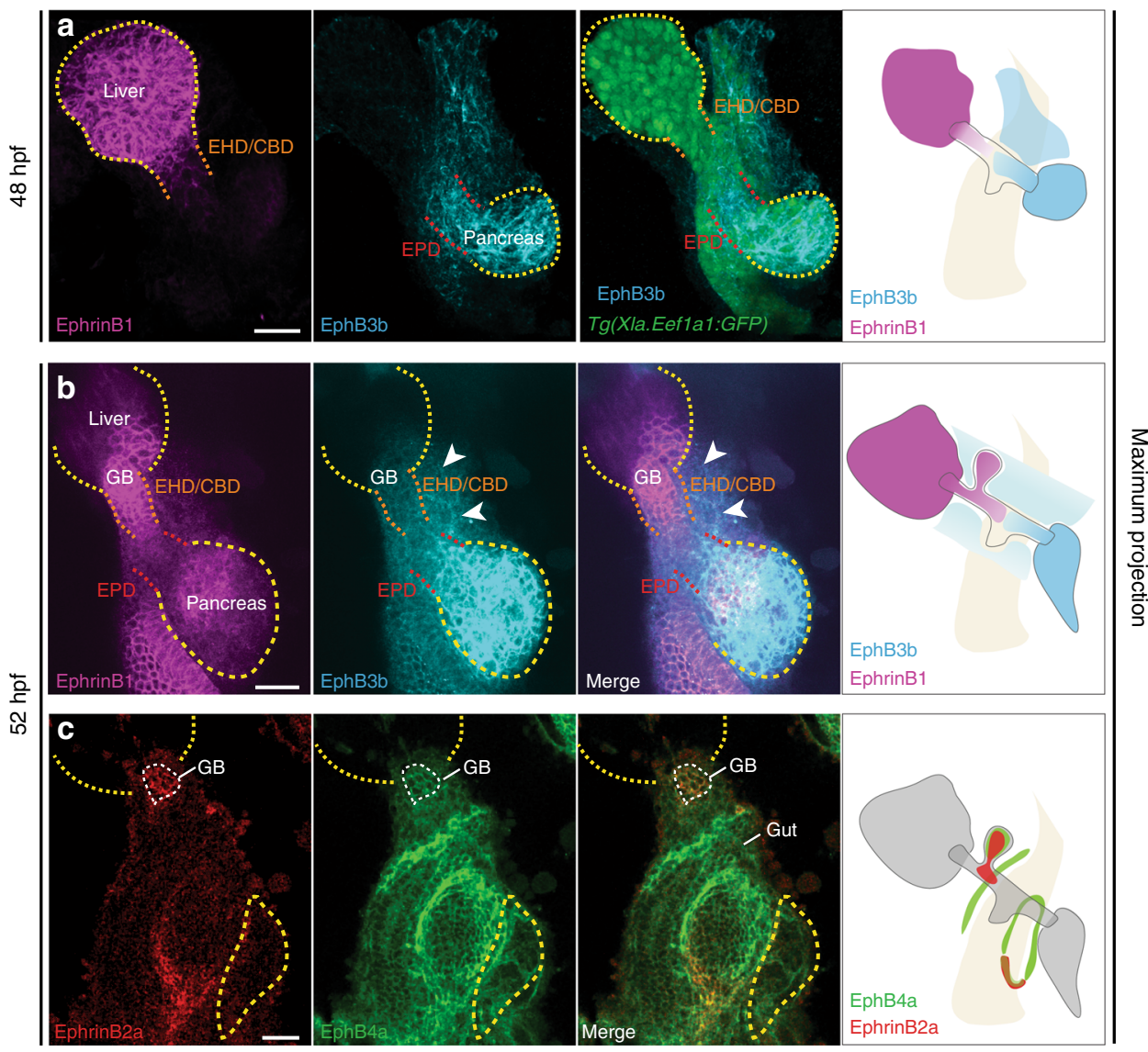

Fig. 6 Regionalized HPD endoderm and mesoderm expression of multiple EphrinBs and EphBs. a At 48 hpf, EphrinB1 is strongly expressed in the liver and at lower levels in the HPD primordium close to the liver. EphB3b expression is high in the pancreas and in the HPD primordium close to the pancreas and lower close to the liver, as well as very low in the HPD-adjacent mesoderm; pan-endodermal tg(Xla.Eef1a1:GFP) highlights the digestive system. b At 52 hpf, EphrinB1 is expressed in the liver (yellow dotted line) and EHB (white arrow), while EphB3b is highly expressed in the pancreas (yellow dotted line) and at low levels in the EPD (white arrow) and HPD mesenchyme (white arrowheads). c EphrinB2a and EphB4a are co-expressed in the gallbladder (white dashed line). EphrinB2a is present in the posterior region of the swim bladder. EphB4a is in the mesenchyme along the gut tube and around the swim bladder. Scale bar $=30 \mu \mathrm{m}$

and at lower levels in the HPD primordium next to the liver, whereas EphB3b shows strong expression in the pancreas and HPD adjacent to the pancreas and weak expression adjacent to the liver and HPD-surrounding mesenchyme (Fig. 6a). At $52 \mathrm{hpf}$ and as the HPD starts differentiating, EphrinB1 is expressed throughout the liver and in the EHD, GB, CBD and intestinal epithelium, while EphB3b is expressed highly in the pancreas and at lower levels in the EPD and mesoderm surrounding the HPD system (Fig. 6b). Given their bidirectional signaling capabilities, this expression profile suggests that EphrinB1 may interact with EphB3 at (i) the endoderm-mesoderm interface for the EHD, GB and $\mathrm{CBD}$ and (ii) within the duct at the CBD-EPD boundary (Fig. 6b). Notably, EphrinB1 expression in ephb3b mutants expands from the CBD into the EPD (Supplementary Fig. 7), possibly because of the absence of EphB3b-mediated membrane removal by endocytosis ${ }^{38}$. Alternatively, EPD cells express EphrinB1 due to the release of repressive EphB3b effectors at the transcriptional level.

Next, we investigated EphrinB2a and EphB4a as additional regulators of HPD formation, since they frequently act in concert with EphrinB1 and/or EphB3 in complex morphogenetic processes $^{21}$, such as vascular morphogenesis ${ }^{39}$, cell positioning in the intestine $e^{40}$ or tissue separation ${ }^{27,29}$. To assess their spatiotemporal expression, we generated polyclonal antibodies revealing EphrinB2a expression in the GB primordium and at lower levels in adjacent parts of the CBD at $52 \mathrm{hpf}$, while EphB4a is co-expressed in the GB and non-HPD mesoderm (Fig. 6c). Hence, these four genes are expressed in a regionalized and partly overlapping pattern during HPD development and in differentiated ducts. In addition, mammalian conservation was suggested by expression analysis of human tissue samples, revealing EPHRINB1, EPHRINB2, and EPHB4 expression in large biliary ducts and EPHRINB1, EPHRINB2, EPHB3, and $E P H B 4$ in extrahepatic cholangiocarcinoma tissue, serving as closest avatar, since access to healthy tissue is difficult (Supplementary Fig. 8).

To assess EphrinB2a and EphB4a functions in HPD development, we analyzed mutants for HPD lumen formation by aPKC staining at $60 \mathrm{hpf}$ and HPD morphology by Anxa4 staining at 5 dpf. ephrinb2a mutants exhibit unresolved loop-like lumina, both in the GB and CBD, at $60 \mathrm{hpf}$ (Fig. 7c, f, Supplementary Movie 5), and substantially smaller GBs and dysmorphic CD and CBD at 5 dpf (Fig. 7g, i, Supplementary Movie 11). Given the EphrinB1 and EphrinB2a co-expression in overlapping HPD domains, we assessed their genetic relationship in double mutants. Most ephrinb1;ephrinb2a HPD phenotypes are more severe, including cysts in the EHD, GB and CBD region at $60 \mathrm{hpf}$, consistent with additive phenotypes (Fig. 7b-d, f, Supplementary Movie 6). Similarly at $5 \mathrm{dpf}$, ephrinb1; ephrinb2a double mutants display significantly smaller GBs and short dysmorphic EHDs and CBDs (Fig. 7h-j, l, m, Supplementary Movie 12). ephb4a mutants showed a transiently smaller GB primordium compared 

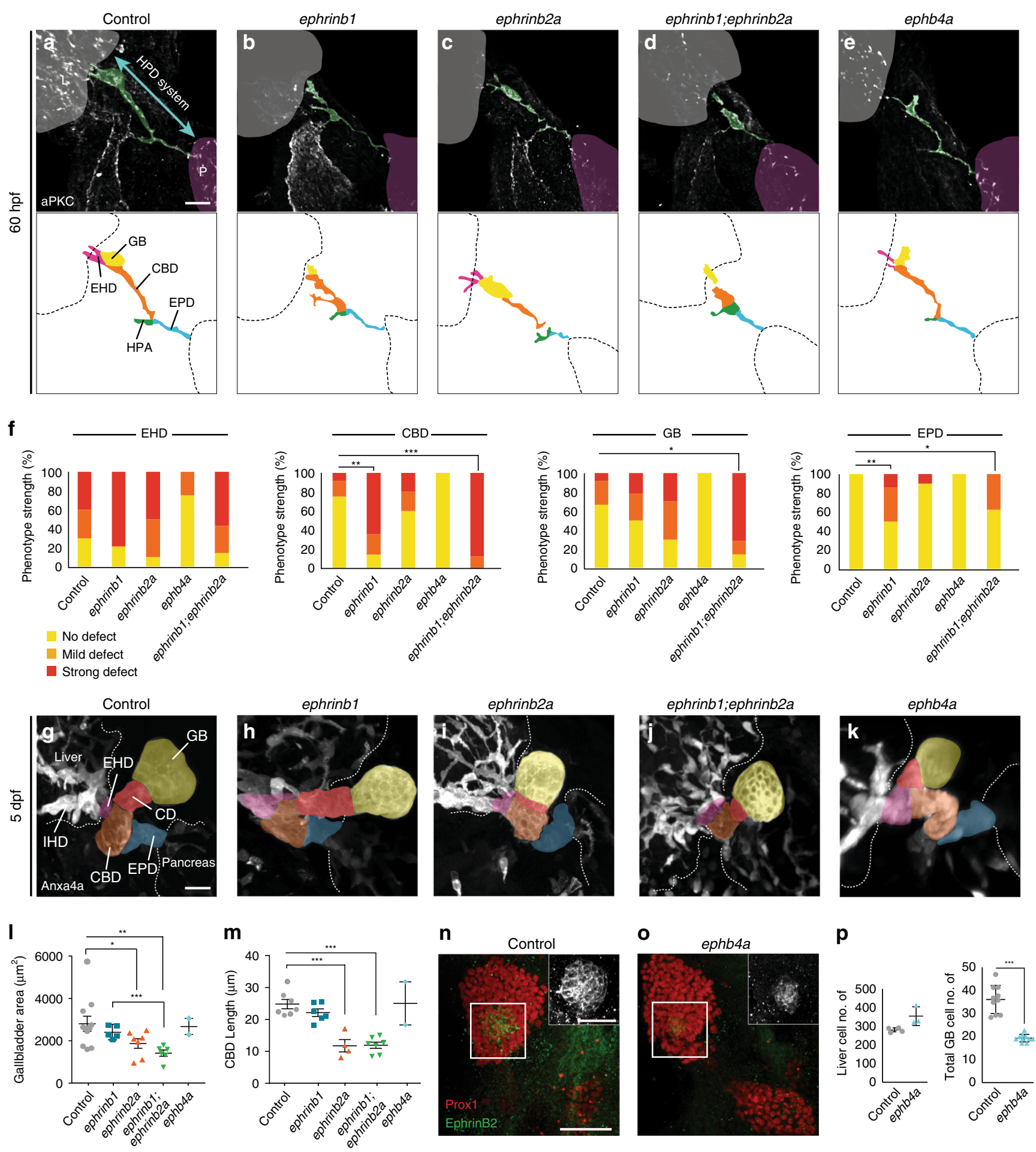

Fig. 7 EphrinB2a and EphB4 functions in gallbladder and CBD formation. Unlike sibling controls (a), ephrinb1 mutants (b) show disrupted ducts in the EHB region. ephrinb2a mutants (c) exhibit dysmorphic gallbladder and CBD (46.1\% show the full phenotype, 53.9\% show milder phenotype, $n=13, N=2)$. d ephrinb1; ephrinb2a double mutants show additive EHB duct defect $(100 \% n=8, N=1)$. e ephb4a mutants show no apparent HPD defect $(n=4, N=1)$. Schematics of immature HPD lumina show in a-e in domain-specific color code. f Quantification of ephrinb1, ephrinb2a, ephb4a and ephrinb1; ephrinb2a double mutant HPD formation defects at 60 hpf, scored for individual HPD domains (control: $n=12$; ephrinb1: $n=14$; ephrinb2a: $n=10$; ephb4a: $n=4$, ephrinb1; ephrinb2a: $n=8$ ); see Supplementary Fig. 3 for averaged scores from two assessors. g-k Early duct morphogenesis defects cause dysmorphic HPD appearance at $5 \mathrm{dpf}$ in ephrinb1, ephrinb2a and ephrinb1;ephrinb2a double mutants, while the HPD in ephb4a mutants appears control-like. (control: $n=10$, $N=2$; ephrinb1: $n=6, N=1$; ephrinb2a: $n=7$; ephrinb1;ephrinb2a: $n=6$; ephb4a: $n=2$ ) I Area quantification shows the gallbladder is significantly smaller in ephrinb1; ephrinb2a double mutants at $5 \mathrm{dpf}$. $\mathbf{m}$ Quantification of CBD length at $5 \mathrm{dpf}$ shows significant differences in ephrinb2a and ephrinb1;ephrinb2a mutants. n-p In contrast to controls (n), fewer EphrinB2a-positive gallbladder precursors form in ephb4a mutants at $52 \mathrm{hpf}(\mathbf{0})$. $\mathbf{p}$ Quantification of liver and gallbladder progenitor numbers (control and ephb4a $n=10$ ). a-e: Scale bar $=15 \mu \mathrm{m}, \mathbf{g}-\mathbf{k}=20 \mu \mathrm{m}$. Statistical test: $\mathbf{f}=$ Fisher Exact, $\mathbf{I}, \mathbf{m}, \mathbf{p}=$ Student's $t$ test. Error bars show SEM; ${ }^{\star} p<0,05 ;{ }^{* \star} p<0,01 ;{ }^{\star \star \star} p<0,001$. Supplementary movies 1, 2, 5-9, 11-13 show HPD phenotypes at 60 hpf and 5 dpf. Source data are provided as a Source Data file 
to unchanged liver progenitor numbers at $52 \mathrm{hpf}$ (Fig. $7 \mathrm{n}-\mathrm{q})$, but no detectable change in the HPD at $60 \mathrm{hpf}$ and 5 dpf (Fig. 7e, f, $\mathrm{k}-\mathrm{m}$, Supplementary Movies 7,13 ), indicating compensation by other factors. Likewise, ephb3b; ephb4a double mutants showed no additional phenotypes to those of ephb3b mutants (data not shown). Together these data demonstrate compartment-specific expression and requirements for EphrinB1, EphrinB2a, EphB3b, and EphB4a in HPD duct remodeling and GB formation, revealing an EphB/EphrinB code-like control of HPD morphogenesis, including signaling interactions at multiple endodermmesoderm and intra-endodermal tissue interfaces.

\section{Discussion}

In this study, we show that the HPD system differentiates by a dynamic morphogenetic process, in which a mature tube forms by a multi-step cord-hollowing mechanism. This includes remodeling from multiple nascent lumina into a single lumen, which requires active cell intercalation and actomyosin contractility. We identify EphB/EphrinB signaling as a key regulator of this step. Specifically, multiple EphB receptors and EphrinB ligands are required for HPD morphogenesis in a compartmentspecific fashion. In light of the regionalized and partly overlapping ligand and receptor expression in the HPD endoderm and surrounding mesoderm we propose that compartmentspecific duct remodeling is controlled by a morphogenetic EphB/ EphrinB code that involves signaling at multiple tissue interfaces (Fig. 8a, b).

The HPD system primordium forms between the outgrowing liver and ventral pancreas buds $2,15,41$ and we show that HPD tubes form concomitant to the differentiating IHDs and IPDs. Polarity acquisition of HPD progenitors is marked by the emergence of multiple tight junction protein ZO-1 aggregates, which subsequently mature into nascent apical $\mathrm{aPKC}^{+}$microlumina. Upon expansion, they connect with adjacent microlumina coalescing into a continuous nascent lumen, transitioning through intermediates of luminal loops and occasionally parallel lumina. Our findings thus demonstrate that HPD de novo lumen formation occurs by a dynamic multi-step process to generate epithelial tubes by cord hollowing (Fig. 8c), similar to lumen morphogenesis in the zebrafish gut ${ }^{42-44}$ and the steps underlying ductal plexus formation in the mouse pancreas $32,33,45$.

Our high-resolution analysis further revealed that cell intercalation is part of the cell rearrangements transforming the multilayered cord into a single-layered ductal epithelium. HPD teardrop-shaped cells extend apical filopodia during lumen remodeling similar to radial intercalation of multi-ciliated cells into the epidermis in Xenopus ${ }^{46}$ and extending mammary gland ductules ${ }^{35}$ corroborating the role of cell intercalation in driving epithelial tube elongation, such as Drosophila trachea or in the vertebrate kidney 47,48 , whereby the exact mode (e.g., radial, rosette mediated) may differ.

Adding to the mechanistic understanding of cord hollowing, we demonstrate the importance of actomyosin contractility. Given the diverse roles of myosin II in morphogenesis, it is likely involved in multiple steps of tubulogenesis. First, apical contraction by actomyosin activity controls lumen size ${ }^{49,50}$, while inhibition leads to cell spreading. Controlled apical actomyosin contractility may allow just enough cell spreading to ensure fusion of nascent microlumina, while loss of contractility leads to excessive lumen expansion. In line with this, HPD progenitors exhibit high apical pMLC and blocking myosin II during CBD lumen resolution results in an expanded luminal network. Conversely, an increase of apical pMLC levels in ephrinb1 mutant CBDs is associated with an increase of teardrop-shaped cells and domain shortening. This suggests that EphrinB1 promotes the

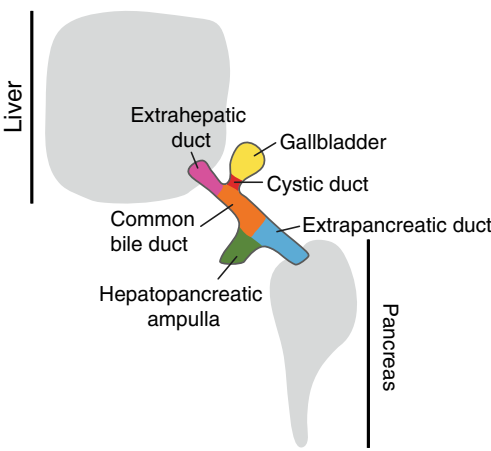

a
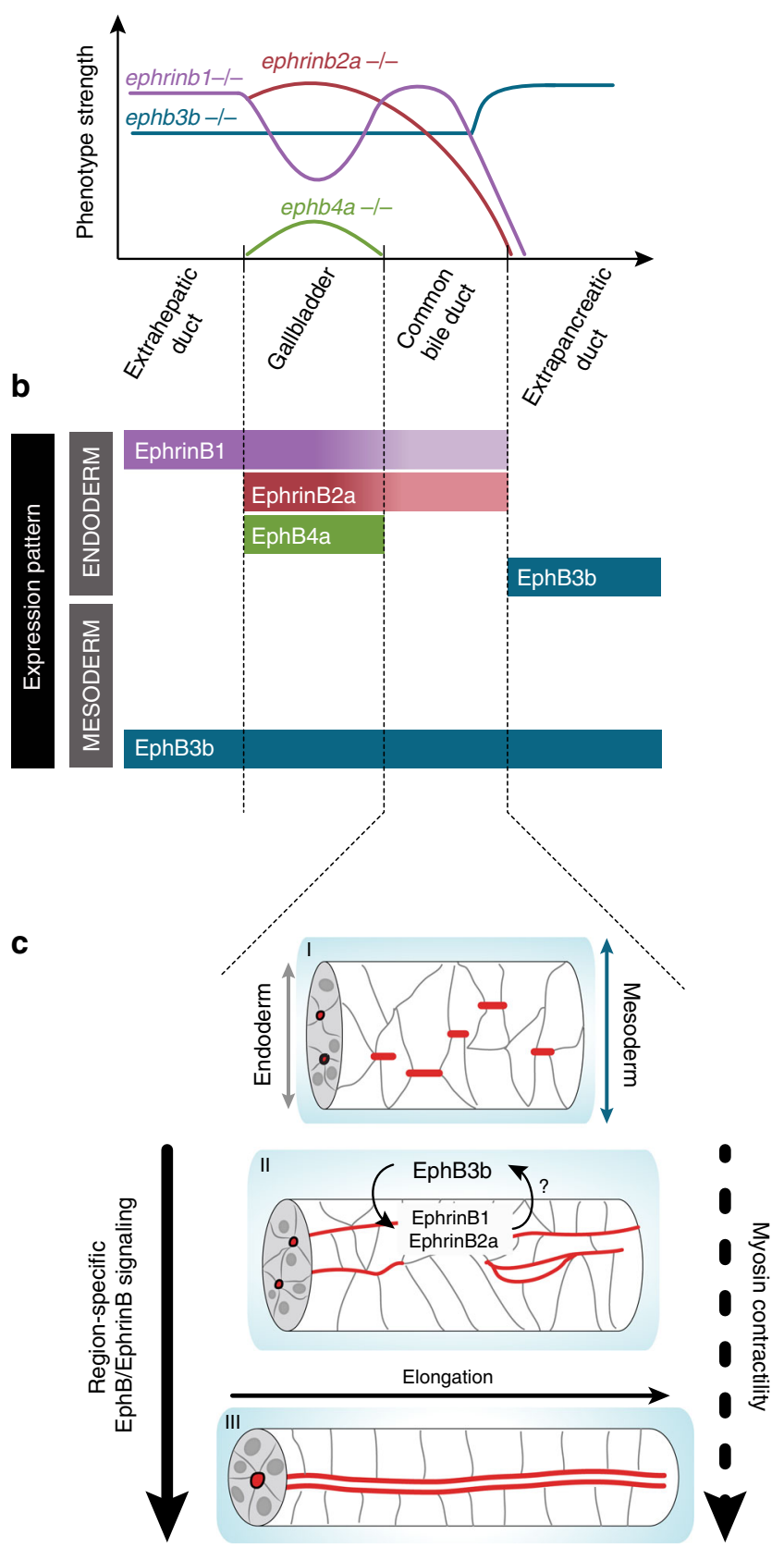

acquisition of distinct apical myosin II levels, which prevent cell spreading and thereby ensure moderate contractility critical for coordinated cell intercalation and balanced apical expansion during CBD formation. We thus propose that tight spatial and temporal control of apical membrane size is essential for lumen coalescence in cord hollowing. Constraining lumen size by 
Fig. 8 An EphB/EphrinB code and myosin II contractility control HPD tubulogenesis. a Graphic representation of domain-specific phenotypes observed in ephrinb1, ephrinb2a, ephb3b and ephb4a mutants; $y$-axis indicates overall severity of phenotypes. $\mathbf{b}$ Graphic representation of domain-specific expression of EphrinB1, EphrinB2a, EphB3b and EphB4a in HPD endoderm and surrounding mesoderm at $52 \mathrm{hpf}$. c Working model of HPD de novo lumen formation focused on the CBD domain. Lumen formation occurs by a multi-step cord hollowing mechanism, which starts with scattered junctional aggregates, transitions through apical/luminal pocket formation and their subsequent coalescence and finally resolves into a single lumen. Apical/luminal pocket coalescence is promoted by ductal EphrinB1/ EphrinB2a interaction with mesodermal EphB3b at the tissue interface and actomyosin contractility

myosin II is important during ascidian notochord formation ${ }^{51}$, lumen expansion in zebrafish and murine blood vessel development ${ }^{49,50}$ and branching of pancreatic ducts ${ }^{52}$. Furthermore, blocking myosin II and its localization to actin fibers at the leading edge of migrating cells ${ }^{53}$ would compromise cell intercalation and hence formation of a single-layered epithelial duct. Finally, low pMLC at the lateral side of CBD cells indicates that it may control membrane shortening, similar to ascidian endoderm invagination ${ }^{54}$. Altogether, our data show a mechanistic link between EphrinB1 and myosin II pathway. EphrinB1 could directly control apical pMLC localization, or indirectly, for instance via RhoA and ROCK, known effectors of EphB/EphrinB signaling ${ }^{20}$. Co-expression of EphrinB1 and EphrinB2a combined with additive CBD defects in double mutants point to similar functions for EphrinB2a in this process. Milder cell shape defects in $e p h b 3 b$ CBDs suggest that EphB3b controls tubulogenesis only partly by EphrinB-mediated apical pMLC levels. EphB/EphrinB signaling could further contribute to other roles in cell migration/ intercalation and/or basolateral membrane shortening during HPD tube formation. The importance of cytoskeletal remodeling in biliary ducts is highlighted by ductal defects in zebrafish mutants for Hippo-Yap pathway component Nf2 and the serinethreonine kinase $\mathrm{Cdk} 5^{55,56}$

Repulsion-based activity of regionalized Ephs and Ephrins is known to coordinate complex morphogenetic developmental processes, such as rhombomere boundary formation in the hindbrain ${ }^{27}$ or axon guidance to generate topographic maps in the retinotectal system ${ }^{21,57}$. During early organogenesis, EphBs and EphrinBs direct the asymmetric migration of hepatoblasts to their final position ${ }^{30}$. Yet for the HPD system, we show that regionalized EphB and EphrinB activity is required for compartment-specific tube morphogenesis (Fig. 8a). Both the spatiotemporal expression and mutant phenotypes suggest that EphB/EphrinB signaling regulates HPD morphogenesis from the onset, and early progenitor positioning may partly contribute. This uncovers a putatively more general role of Eph/Ephrin signaling in directing tube morphogenesis in complex ductal systems, because regionalized expression is reported in the mouse kidney ${ }^{58}$. Specifically, EphB3b is required for the remodeling of microlumina into a single lumen throughout the HPD, while EphrinB1 controls only the remodeling of the EHB, but not the EPD. EphB3b therefore interacts with an additional currently unknown EphrinB ligand in EPD morphogenesis, or acts in a ligand-independent fashion ${ }^{59}$. EphrinB2a and EphB4a regulate GB size, either by mediating progenitor specification, aggregation of GB progenitors into the prospective GB primordium or by proliferation. Therefore, regionalized members of the same gene family ensure efficient and coordinated morphogenesis of all HPD parts, resulting in compartment-specific morphology of the individual ducts. First insights of how these complex expression patterns arise, come from analysis of $s o x 9 b$ mutants exhibiting a mis-patterned and dysmorphic HPD system ${ }^{15,16}$. We show that EphrinB1 expression in the CBD is reduced in $60 \%$ of $s o x 9 b$ mutants (data not shown), indicating that Sox9b controls HPD patterning and morphogenesis via independent effectors.

Given the bidirectional signaling capabilities of EphB/EphrinB signaling, the regionalized expression of two EphrinB ligands and two EphB receptors during HPD development allows different signaling possibilities. EphrinB1/EphrinB2a expression in all EHB compartments, and EphB3b expression in the EHD surrounding mesoderm and the EPD endoderm (Fig. 8b), suggests signaling in trans across two receptor-ligand interfaces: (i) remodeling of the EHB ducts can be mediated by signaling across the endodermmesoderm boundary, with EphrinB1/EphrinB2a reverse signaling controlling the dynamic cell rearrangements in the ductal epithelium. (ii) EphrinB1/EphB3b interactions at the CBD-EPD boundary may regulate duct remodeling via reverse signaling in the EphrinB1-positive CBD, and by forward signaling in the EphB3b bearing EPD. This disruption of the CBD-EPD junction is similar to pancreaticobiliary maljunctions of patients with twoway reflux of bile and pancreatic juice, inflammation and metaplasia of ductal cells ${ }^{60}$. Notably, ductal tight junction proteins and myosin light chain kinase are altered in these cases, corroborating the myosin II requirement for HPD formation shown in our study. The additive EHB defects in ephrinb1;ephrinb2a double mutants indicate that both ligands interact with mesodermal EphB3b, reflecting increased signaling strength, similar to what has been observed in Xenopus germ layer separation ${ }^{29}$ and in line with promiscuous ligand-receptor interactions ${ }^{20}$.

Blocking EphB/EphrinB signaling by dominant negative EphrinB1 ${ }^{\mathrm{EC}}$ expression, demonstrates that HPD lumen resolution is specific and independent from earlier EphB/EphrinB functions in left-right signaling or asymmetric liver progenitor positioning ${ }^{30,61}$, revealing temporally distinct sequential roles in organogenesis. Finally, the expression of EPHBs and EPHRINBs in human indicates a conserved role in epithelial maintenance of large biliary ducts and possible HPD morphogenesis across vertebrates.

This systematic analysis describes how functional HPD tubes form to connect the liver and pancreas to the intestine, establishing a reference for future studies of HPD development and to further our understanding of congenital cases of HPD malformation. The identification of regionalized activity of multiple EphrinB ligands and EphB receptors uncovers a paradigm for coordinating the morphogenesis of multi-compartment ductal systems and subsequent maintenance of tissue integrity.

\section{Methods}

Zebrafish stocks. Adult zebrafish and embryos (Danio rerio) were kept and raised according to standard laboratory conditions ${ }^{62}$. All experiments were performed according to ethical guidelines approved by the Danish Animal Experiments Inspectorate (Dyreforsøgstilsynet) or the UK Home Office under the Animals (Scientific Procedures) Act 1986. The following strains were used: $\mathrm{Tg}$ (UAS: ephrinb1 $\left.{ }^{E C}\right)^{n i m} 25$ 30, $\mathrm{Tg}$ (hsp70l:Gal4)fil (gift from David Wilkinson), ephrinb1 ${ }^{\text {nim } 2630}$, ephrinb2ahu3393 and ephb4ahu3378 (Stemple Laboratory, direct submission to ZFIN), Tg(keratin18:GFP)p31463 and Tg(Xla.Eef1a1:GFP)s854 41 .

Generation of genetic mutants. The ephb3b nim27 mutant allele was generated by CRISPR/Cas9 injections into one-cell-stage zebrafish embryos ${ }^{64}$. A single sgRNA targeting the sequence $5^{\prime}$-GGG CCA TGA CTG AGC TGG CC-3" in the second exon of EphB3b (Supplementary Fig. 1) was created by annealing and cloning two complementary oligonucleotides into the pDR274 backbone (Addgene \#42250). In vitro synthesis of the sgRNA was performed using the T7 RiboMAX ${ }^{\mathrm{TM}}$ Large Scale RNA Production System (Promega). Mutations were analyzed by amplicon restriction using the primers ephb3bF $5^{\prime}$-GGCCTGTTGTTTCCTCAGACT-3' and $e p h b 3 b \mathrm{R} 5^{\prime}$-GCAAAACATGACCTTGTTGA-3', followed by XcmI restriction and verified by sequencing. A carrier fish transmitting a 4 bp deletion affecting the ligand-binding domain was used to generate a stable line. 
Table 2 PCR and restriction digest information for mutant genotyping

\begin{tabular}{|c|c|c|c|c|}
\hline Mutant & Forward primer & Reverse primer & $\begin{array}{l}\text { Annealing } \\
\text { temperature }\end{array}$ & Restriction enzyme \\
\hline ephrinb1 & 5'-GTTTGTGTCTGGGAAGGGCTTAG-3' & 5'-TATGGTGCTGCAGGACTCGGCCTG-3' & $65^{\circ} \mathrm{C}$ & $\begin{array}{l}\text { Xcml } \\
\text { (mutant: } 2 \text { fragments) }\end{array}$ \\
\hline ephrinb2a & 5'- TTTTGATCTAGAGAGAAATGCGAGT-3' & 5'- TAGAGGCGTGTCTGCTTTTGACACCTG-3' & $53^{\circ} \mathrm{C}$ & $\begin{array}{l}\text { PshAl } \\
\text { (mutant: } 2 \text { fragments) }\end{array}$ \\
\hline
\end{tabular}

Genotyping of genetic mutants. Adult mutant carriers are identified by PCR and restriction digest-based genotyping of tailfin tissue. To genotype embryos for experiments, we collected 1-2 $\mathrm{mm}$ of tail tissue after fixation to determine the genetic status by PCR and restriction digest. Conditions for PCR and restriction digests are shown in Table 2.

Immunohistochemistry. Embryos are fixed with $4 \%$ paraformaldehyde (PFA) overnight at $4{ }^{\circ} \mathrm{C}$, permeabilized with PBST (PBS with $0.1 \%$ Triton $\mathrm{x}-100$ ) and deyolked, followed by blocking with $1 \%$ Triton- 100 and $10 \%$ donkey/goat serum in PBS for $1 \mathrm{~h}$ and incubated with primary antibodies, $\alpha-2 \mathrm{~F} 11$ (mouse monoclonal, 1:1000, gift from Julian Lewis), $\alpha$-ZO1 (mouse monoclonal antibody, 1:200, Invitrogen, 33-9100), aPKCל (rabbit polyclonal antibody,1:1000, Santa Cruz, sc-216), $\alpha$-pan-Cadherin (mouse monoclonal antibody, 1:1000, Sigma, C1821, rabbit $\alpha$ EphrinB $1^{30}$ and guinea pig $\alpha$-EphB3b $\mathrm{b}^{30}$ overnight at $4{ }^{\circ} \mathrm{C}$. After repeated washing with PBST, embryos are incubated in secondary antibodies and fluorescent stains, donkey-anti-rabbit Cy3 (1:200, Jackson Immunoresearch, 112-166-143), donkeyanti-mouse 647 (1:400, Jackson Immunoresearch, 715-605-151), donkey-anti-goat 488 (1:200, Jackson Immunoresearch, 705-545-147) and Alexa Fluor 488 Phalloidin (1:500, Molecular Probes, A12379), Alexa Fluor 647 Phalloidin (1:500, Molecular Probes, A22287), DAPI (1:1000, Sigma, D9542) overnight at $4{ }^{\circ} \mathrm{C}$. For nuclear protein detection with $\alpha$-Prox1 (mouse monoclonal antibody, 1:50, Abcam, ab33219), rabbit polyclonal antibody, 1:500, Angiobio, 11-002P) and $\alpha$-Hnf4a (goat polyclonal antibody, 1:100, Santa Cruz, sc-6556), embryos are incubated with DNaseI enzyme at $37^{\circ} \mathrm{C}$ for 45 min prior to blocking 65 and washed with PBS, followed by normal staining protocol. To detect phospho-MLC we followed Sidhaye et al. ${ }^{66}$, we permeabilized embryos with PBST (PBS with $0.8 \%$ Triton X-100) and trypsinized on ice for $15 \mathrm{~min}$, followed by blocking with $10 \%$ goat serum and incubation in a-pMLC (rabbit Ser19; 1:100; Cell Signaling, \#3671S) 1\% goat serum in PBST for $60 \mathrm{~h}$ at $4{ }^{\circ} \mathrm{C}$. After washing, the secondary antibody mix with $1 \%$ goat serum in PBST is added for $60 \mathrm{~h}$.

Polyclonal antibodies against EphrinB2a and EphB4a were generated in rabbits and guinea pigs, respectively ${ }^{30}$. We subcloned the ectodomain region with the native signal peptide of zebrafish EphrinB2a and EphB4a into a mammalian expression vector containing a C-terminal rat $\mathrm{Cd} 4 \mathrm{~d} 3+4$ tag and a hexa-his tag for expression of a soluble recombinant protein by transient transfections into HEK293E cells (Bushell et al., 2008). $\mathrm{Ni}^{2+}$-affinity chromatography (GE Healthcare) was used for protein purification. The animal facility contracted to produce antibodies is based in the UK and governed by UK legislation including the Animal (Scientific Procedures) act 1986 . This facility was regularly inspected by the UK Home Office and all works subject to an ethical review.

Embryos were mounted either in 4\% agarose and sectioned $130 \mu \mathrm{m}$ using a Leica Vibratome or as whole-mount in Benzyl Alcohol, Benzyl Benzoate (BABB) clearing mixture prior to imaging. The embryos were imaged with a Leica SP8 or a Zeiss 780 confocal microscope. Images were processed with Imaris (Bitplane) image analysis software and Adobe Photoshop CS.

Heat-shock experiment. Embryos collected from $\operatorname{Tg}\left(\text { UAS: ephrinb } 1^{E C}\right)^{n i m} 25$ and $\mathrm{Tg}(\mathrm{hsp} 70 \mathrm{l}: \mathrm{Gal} 4)^{f c i l}$ intercrosses were subjected to heat shocks at $39^{\circ} \mathrm{C}: 45 \mathrm{~min}$ either at $50 \mathrm{hpf}$ or $54 \mathrm{hpf}$. The embryos were then returned to $28^{\circ} \mathrm{C}$ incubator until fixation at $60 \mathrm{hpf}$.

Mosaic cell labeling by DNA injection. Membrane-targeted lyn-tdTomato under the control of the ubiquitin (ubi) promoter was co-injected at 20-30 pg DNA/ embryo with 30-80 pg/embryo transposase mRNA into 1-cell-stage Tg(Xla.Eef1a1: GFP) $)^{s 54}$ embryos.

Blebbistatin treatment. Myosin II ATPase activity was inhibited by blebbistatin ${ }^{67}$. Considering the impact of actomyosin dependent contractility in various cellular processes, we used mild doses of the drug $(65 \mu \mathrm{M}$ of final concentration in embryo water). Embryos were incubated from $52 \mathrm{hpf}$ until $60 \mathrm{hpf}$ at $28^{\circ} \mathrm{C}$ under slow shaking conditions to prevent precipitation of the solution. This treatment caused no apparent change to the overall embryo body phenotype.
Quantification of phenotype severity. The variability of the mutant lumen formation defects was quantified by scoring ductal malformations in a domainspecific fashion. Each domain of the HPD system was assessed individually on a scale from 0 to 2, with $0=$ no defect; $1=$ mild defect; $2=$ strong defect (Supplementary Fig. 3a-f, Table 1). 3D-confocal stacks of aPKC immunostainings of control and mutant HPDs were renamed for blind analysis and independently scored by two authors. The scores were independently analyzed with the Fisher Exact Test for frequency data. The robustness of the scoring was assessed by comparing and averaging both sets of scores. Given they are frequency data, they were first converted into ordinal data and then tested with the Wilcoxon test for non-parametric data (Supplementary Fig. 3g-j).

Cell shape analysis. To assess CBD cell shapes, the length of apical and basal cell domains and cell height were measured in embryos, in which aPKC labeled the apical membrane, panCadherin outlined cells and nuclear Dapi served to identify individual cells. 3D-stacks of confocal images were processed in 2D focal sections with Imaris (Bitplane) analysis software and cellular parameters were determined with the Measurement plug-in. Cells with unclear boundaries or complex membrane topologies possible due to contributions from multiple cells were excluded from the analysis. Statistical significance was assessed by unpaired Student's $t$-test.

EdU incorporation assay and proliferation analysis. Embryos were incubated for $60 \mathrm{~min}$ at $28.5^{\circ} \mathrm{C}$ in $400 \mu \mathrm{M}$ 5-ethynyl-2'-deoxyuridine (EdU) with $15 \%$ DMSO in egg water, and control larvae were treated with 15\% DMSO. Larvae were fixed after incubation for $90 \mathrm{~min}$ at room temperature and processed using the Click-iT EdU Imaging Kit (Invitrogen). EdU incorporation was quantified with Imaris analysis software. Anxa4 antibody staining was used to mask the HPD ducts via the surface tool. DAPI- and EdU-positive cells were counted in the masked channels by the spot function and the ratio of EdU- to DAPI-positive cells was calculated subsequently.

Quantification of pMLC levels. Wild-type and ephrinb1 mutant embryos stained for pMLC, ZO-1 and actin were used for pMLC quantification. Using FIJI ${ }^{68}$, for each sample three linear ROIs where drawn perpendicular to the duct luminal axis and on a single plane, in which the lumen was clearly visible; each ROI was 10 pixels $/ 1.9 \mu \mathrm{m}$ wide and spanning the whole tube across opposite basal sides $(52.7 \mu \mathrm{m})$. Average intensities in each position of the line were extracted using the Plot Profile plugin in FIJI. A rectangular ROI of $40 \mu^{2}$ was used to extract the average background intensity in each channel and used to normalize the fluorescence intensity values within the linear ROIs. Two regions of $2 \mu \mathrm{m}$ each were selected across the apical peaks in the intensity profile of the ZO- 1 channel and the background-corrected pMLC values where integrated in the chosen areas for each linear ROI (see Supplementary Fig. 5).

Microarray analyses of human tissue samples. Preprocessing of microarray data from LEC2012 ${ }^{69}$, LEC2018 and normal bile duct (NBD) include background correction, quantile normalization and $\log _{2}$-transformation of intensity values. Differentially expressed genes were calculated by $\mathrm{t}$-test analysis using R v3.3. All samples were obtained with approval by the institutional review board of the National Institutes of Health and collaborating institutions on the condition that patients were anonymized ${ }^{69}$ and subsequently approved by the Danish ethics committee.

Reporting summary. Further information on research design is available in the Nature Research Reporting Summary linked to this article.

\section{Data availability}

The authors declare that all data supporting the findings of this study are available within the article and its supplementary information files or from the corresponding author upon reasonable request. The image data generated and analyzed in this study are available from the corresponding author upon reasonable request. A source data file for 
the quantifications shown in Figs. 1g, 2h, o, p, 3e-g, 4e, h, 7f, 1, m, p and Supplementary Figs. $3 \mathrm{~g}-\mathrm{j}$ and $6 \mathrm{~d}$, e are provided as a Source Data file.

Received: 8 February 2019; Accepted: 18 October 2019; Published online: 19 November 2019

\section{References}

1. Villasenor, A. \& Stainier, D. Y. R. On the development of the hepatopancreatic ductal system. Seminars Cell Developmental Biol. 1-12 (2017). https://doi.org/ 10.1016/j.semcdb.2017.02.003

2. Dong, P. D. S. et al. Fgf10 regulates hepatopancreatic ductal system patterning and differentiation. Nat. Genet. 39, 397-402 (2007).

3. Zhao, X. et al. Glutathione antioxidant pathway activity and reserve determine toxicity and specificity of the biliary toxin biliatresone in zebrafish. Hepatology 64, 894-907 (2016)

4. Lakshminarayanan, B. \& Davenport, M. Biliary atresia: a comprehensive review. J. Autoimmun. 73, 1-9 (2016).

5. Waisbourd-Zinman, O. et al. The toxin biliatresone causes mouse extrahepatic cholangiocyte damage and fibrosis through decreased glutathione and SOX17. Hepatology 64, 880-893 (2016).

6. Tadokoro, H., Takase, M. \& Nobukawa, B. Development and congenital anomalies of the pancreas. Anat. Res. Int. 2011, 351217-351217 (2011).

7. Kamisawa, T. et al. Pancreaticobiliary Maljunction. YJCGH 7, S84-S88 (2009).

8. Spence, J. R. et al. Sox17 Regulates Organ Lineage Segregation of Ventral Foregut Progenitor Cells. Dev. Cell 17, 62-74 (2009).

9. Hunter, M. P. et al. The homeobox gene Hhex is essential for proper hepatoblast differentiation and bile duct morphogenesis. Dev. Biol. $\mathbf{3 0 8}$ 355-367 (2007).

10. Sumazaki, R. et al. Conversion of biliary system to pancreatic tissue in Hes1deficient mice. Nat. Genet. 36, 83-87 (2004).

11. Clotman, F. et al. The onecut transcription factor HNF6 is required for normal development of the biliary tract. Development 129, 1819-1828 (2002).

12. Coffinier, C. et al. Bile system morphogenesis defects and liver dysfunction upon targeted deletion of HNF1beta. Development 129, 1829-1838 (2002).

13. Fukuda, A. et al. Ectopic pancreas formation in Hes1 -knockout mice reveals plasticity of endodermal progenitors of the gut, bile duct, and pancreas. J. Clin. Invest. 116, 1484-1493 (2006).

14. Gao, C. et al. Zebrafish hhex-null mutant develops an intrahepatic intestinal tube due to de-repression of cdxlband pdx1. J. Mol. Cell Biol. 11, e0146806-e0146815 (2018).

15. Manfroid, I. et al. Zebrafish sox9b is crucial for hepatopancreatic duct development and pancreatic endocrine cell regeneration. Dev. Biol. 366, 268-278 (2012).

16. Delous, M. et al. sox9b Is a Key Regulator of Pancreaticobiliary Ductal System Development. PLoS Genet. 8, e1002754-16 (2012).

17. Zhang, D. et al. Identification of Annexin A4 as a hepatopancreas factor involved in liver cell survival. Dev. Biol. 395, 96-110 (2014).

18. Ober, E. A. \& Lemaigre, F. P. Development of the liver: insights into organ and tissue morphogenesis. J. Hepatol. 68, 1049-1062 (2018).

19. Tan, C. E. \& Moscoso, G. J. The developing human biliary system at the porta hepatis level between 29 days and 8 weeks of gestation: a way to understanding biliary atresia. Part 1. Pathol. Int. 44, 587-599 (1994).

20. Pasquale, E. B. Developmental Cell Biology: Eph receptor signalling casts a wide net on cell behaviour. Nat. Rev. Mol. Cell Biol. 6, $462-475$ (2005)

21. Kania, A. \& Klein, R. Mechanisms of ephrin-Eph signalling in development, physiology and disease. Nat. Rev. Mol. Cell Biol. 17, 240-256 (2016).

22. Niethamer, T. K. \& Bush, J. O. Getting direction(s)_ The Eph/ephrin signaling system in cell positioning. Dev. Biol. 1-0 (2018). https://doi.org/10.1016/j. ydbio.2018.01.012

23. Lee, H.-S., Nishanian, T. G., Mood, K., Bong, Y.-S. \& Daar, I. O. EphrinB1 controls cell-cell junctions through the Par polarity complex. Nat. Cell Biol. 10, 979-986 (2008).

24. Park, I. \& Lee, H.-S. EphB/ephrinB signaling in cell adhesion and migration. Mol. Cells 38, 14-19 (2015).

25. Fagotto, F., Rohani, N., Touret, A.-S. \& Li, R. A Molecular Base for Cell Sorting at Embryonic Boundaries: Contact Inhibition of Cadherin Adhesion by Ephrin/Eph-Dependent Contractility. Developmental Cell 27, 72-87 (2013).

26. Jülich, D., Mould, A. P., Koper, E. \& Holley, S. A. Control of extracellular matrix assembly along tissue boundaries via Integrin and Eph/Ephrin signaling. Development 136, 2913-2921 (2009).

27. Cayuso, J., Xu, Q. \& Wilkinson, D. G. Mechanisms of boundary formation by Eph receptor and ephrin signaling. Dev. Biol. 401, 122-131 (2015)

28. Calzolari, S., Terriente, J. \& Pujades, C. Cell segregation in the vertebrate hindbrain relies on actomyosin cables located at the interhombomeric boundaries. EMBO J. 33, 686-701 (2014).
29. Rohani, N., Parmeggiani, A., Winklbauer, R. \& Fagotto, F. Variable combinations of specific ephrin ligand/Eph receptor pairs control embryonic tissue separation. PLoS Biol. 12, e1001955 (2014).

30. Cayuso, J. et al. EphrinB1/EphB3b coordinate bidirectional epithelialmesenchymal interactions controlling liver morphogenesis and laterality. Developmental Cell 39, 316-328 (2016).

31. Baer, M. M., Chanut-Delalande, H. \& Affolter, M. Chapter 6 - Cellular and molecular mechanisms underlying the formation of biological tubes. Essays Dev. Biol. Part B 89, 137-162 (2009). Elsevier Inc.

32. Kesavan, G. et al. Cdc42-mediated tubulogenesis controls cell specification. Cell 139, 791-801 (2009).

33. Villasenor, A., Chong, D. C., Henkemeyer, M. \& Cleaver, O. Epithelial dynamics of pancreatic branching morphogenesis. Development 137 , 4295-4305 (2010)

34. Farber, S. A. et al. Genetic analysis of digestive physiology using fluorescent phospholipid reporters. Science 292, 1385-1388 (2001).

35. Neumann, N. M. et al. Coordination of receptor tyrosine kinase signaling and interfacial tension dynamics drives radial intercalation and tube elongation. Dev. Cell 45, 67-82.e6 (2018).

36. Munjal, A. \& Lecuit, T. Actomyosin networks and tissue morphogenesis. Development 141, 1789-1793 (2014)

37. Holder, N. \& Klein, R. Eph receptors and ephrins: effectors of morphogenesis Development 126, 2033-2044 (1999)

38. Pitulescu, M. E. \& Adams, R. H. Eph/ephrin molecules-a hub for signaling and endocytosis. Genes Dev. 24, 2480-2492 (2010).

39. Adams, R. H. et al. Roles of ephrinB ligands and EphB receptors in cardiovascular development: demarcation of arterial/venous domains, vascular morphogenesis, and sprouting angiogenesis. Genes Dev. 13, 295-306 (1999).

40. Batlle, E. et al. $\beta$-Catenin and TCF mediate cell positioning in the intestinal epithelium by controlling the expression of EphB/EphrinB. CELL 111, 251-263 (2002)

41. Field, H. A., Ober, E. A., Roeser, T. \& Stainier, D. Y. R. Formation of the digestive system in zebrafish. I. Liver morphogenesis. Developmental Biol. 253, 279-290 (2003).

42. Bagnat, M., Cheung, I. D., Mostov, K. E. \& Stainier, D. Y. R. Genetic control of single lumen formation in the zebrafish gut. Nat. Cell Biol. 9, 954-960 (2007).

43. Horne-Badovinac, S. et al. Positional cloning of heart and soul reveals multiple roles for PKC lambda in zebrafish organogenesis. Curr. Biol. 11, 1492-1502 (2001).

44. Alvers, A. L., Ryan, S., Scherz, P. J., Huisken, J. \& Bagnat, M. Single continuous lumen formation in the zebrafish gut is mediated by smootheneddependent tissue remodeling. Development 141, 1110-1119 (2014)

45. Bankaitis, E. D., Bechard, M. E. \& Wright, C. V. E. Feedback control of growth, differentiation, and morphogenesis of pancreatic endocrine progenitors in an epithelial plexus niche. Genes Dev. 29, 2203-2216 (2015).

46. Sedzinski, J., Hannezo, E., Tu, F., Biro, M. \& Wallingford, J. B. Emergence of an Apical Epithelial Cell Surface In Vivo. Dev. Cell 36, 24-35 (2016).

47. Caussinus, E., Colombelli, J. \& Affolter, M. Tip-cell migration controls stalkcell intercalation during Drosophila tracheal tube elongation. Curr. Biol. 18, 1727-1734 (2008)

48. Lienkamp, S. S. et al. Vertebrate kidney tubules elongate using a planar cell polarity-dependent, rosette-based mechanism of convergent extension. Nat. Genet. 44, 1382-1387 (2012).

49. Gebala, V., Collins, R., Geudens, I., Phng, L.-K. \& Gerhardt, H. Blood flow drives lumen formation by inverse membrane blebbing during angiogenesis in vivo. Nat. Cell Biol. 18, 443-450 (2016)

50. Barry, D. M. et al. Rasip1-mediated Rho GTPase signaling regulates blood vessel tubulogenesis via nonmuscle Myosin II. Circ. Res. 119, 810-826 (2016).

51. Denker, E. et al. Regulation by a TGF $\beta$-ROCK-actomyosin axis secures a nonlinear lumen expansion that is essential for tubulogenesis. Development 142, 1639-1650 (2015)

52. Marty-Santos, L. \& Cleaver, O. Pdxl regulates pancreas tubulogenesis and Ecadherin expression. Development 143, 101-112 (2016).

53. Ridley, A. J. Life at the leading edge. Cell 145, 1012-1022 (2011).

54. Sherrard, K., Robin, F., Lemaire, P. \& Munro, E. Sequential activation of apical and basolateral contractility drives ascidian endoderm invagination. Curr. Biol. 20, 1499-1510 (2010).

55. Sadler, K. C., Amsterdam, A., Soroka, C., Boyer, J. \& Hopkins, N. A genetic screen in zebrafish identifies the mutants vps18, nf2 and foie gras as models of liver disease. Development 132, 3561-3572 (2005).

56. Dimri, M. et al. Three-dimensional structural analysis reveals a Cdk5mediated kinase cascade regulating hepatic biliary network branching in zebrafish. Development 144, 2595-2605 (2017).

57. Triplett, J. W. \& Feldheim, D. A. Eph and ephrin signaling in the formation of topographic maps. Semin. Cell Dev. Biol. 23, 7-15 (2012).

58. Weiss, A.-C. \& Kispert, A. Eph/ephrin signaling in the kidney and lower urinary tract. Pediatr. Nephrol. 31, 359-371 (2015).

59. Lisabeth, E. M., Falivelli, G. \& Pasquale, E. B. Eph receptor signaling and ephrins. Cold Spring Harb. Perspect. Biol. 5, a009159-a009159 (2013). 
60. Huang, S.-G. et al. Altered expression levels of occludin, claudin-1 and myosin light chain kinase in the common bile duct of pediatric patients with pancreaticobiliary maljunction. BMC Gastroenterol. 16, 7 (2016).

61. Zhang, J., Jiang, Z., Liu, X. \& Meng, A. Eph/ephrin signaling maintains the boundary of dorsal forerunner cell cluster during morphogenesis of the zebrafish embryonic left-right organizer. Development 143, 2603-2615 (2016).

62. Westerfield, M. A Guide for the Laboratory Use of Zebrafish (Danio rerio). The Zebrafish Book. (University of Oregon Press, Eugene, OR, 2000).

63. Wilkins, B. J., Gong, W. \& Pack, M. A novel keratin18 promoter that drives reporter gene expression in the intrahepatic and extrahepatic biliary system allows isolation of cell-type specific transcripts from zebrafish liver. Gene Expr. Patterns 14, 62-68 (2014).

64. Hwang, W. Y. et al. Efficient genome editing in zebrafish using a CRISPR-Cas system. Nat. Biotechnol. 1-3 (2013). https://doi.org/10.1038/nbt.2501

65. Ober, E. A., Verkade, H., Field, H. A. \& Stainier, D. Y. R. Mesodermal Wnt2b signalling positively regulates liver specification. Nature 442, 688-691 (2006).

66. Sidhaye, J. \& Norden, C. Concerted action of neuroepithelial basal shrinkage and active epithelial migration ensures efficient optic cup morphogenesis. Elife 6, 73 (2017).

67. Straight, A. F. et al. Dissecting temporal and spatial control of cytokinesis with a myosin II Inhibitor. Science 299, 1743-1747 (2003)

68. Schindelin, J. et al. Fiji: an open-source platform for biological-image analysis. Nat. Meth 9, 676-682 (2012).

69. Andersen, J. B. et al. Genomic and genetic characterization of cholangiocarcinoma identifies therapeutic targets for tyrosine kinase inhibitors. Gastroenterology 142, 1021-1031.e15 (2012).

\section{Acknowledgements}

We thank A. Vanoosthuyse for expert technical support, J. Sedzinski, R. Thiagarajan, A. Grapin-Botton, I. Salecker, O. Anderson, and the Ober group for continued discussions or comments on the manuscript, J. Bulkescher and J. Dreyer (DanStem Imaging core facility) for assistance with image acquisition and analysis, and the department of experimental medicine (AEM) for expert fish care. This work was funded by the Novo Nordisk Foundation (NNF17CC0027852) and Danish National Research Foundation (DNRF116). J.C. and D.G. $\mathrm{W}$. were supported by the Francis Crick Institute, which receives its core funding from Cancer Research UK (FC001217), the UK Medical Research Council (FC001217), and the Wellcome Trust (FC001217). S.C. was supported by an SNSF Early Postdoc Mobility fellowship (P2ZHP3_164840) and a Long Term EMBO Postdoc fellowship (ALTF 511-2016), and L.S. and J.B.A. by the Independent Research Fund Denmark (DFF; Sapere Aude2 4183-00118B).

\section{Author contributions}

M.I.T., S.C., and E.A.O. conceived the project. M.I.T., S.C., J.C., R.S.L.H., and R.A performed the experiments and analyzed the data. W.H. performed statistical analysis. L.S. and J.B.A. contributed the human tissue expression data. M.I.T and E.A.O. wrote the manuscript with support from all authors.

\section{Competing interests}

The authors declare no competing interests.

\section{Additional information}

Supplementary information is available for this paper at https://doi.org/10.1038/s41467019-13149-7.

Correspondence and requests for materials should be addressed to E.A.O.

Peer review information Nature Communications thanks Bingcheng Wang, Wolfram Goessling and the other, anonymous, reviewer(s) for their contribution to the peer review of this work. Peer reviewer reports are available.

Reprints and permission information is available at http://www.nature.com/reprints

Publisher's note Springer Nature remains neutral with regard to jurisdictional claims in published maps and institutional affiliations.

(c) (i) Open Access This article is licensed under a Creative Commons Attribution 4.0 International License, which permits use, sharing, adaptation, distribution and reproduction in any medium or format, as long as you give appropriate credit to the original author(s) and the source, provide a link to the Creative Commons license, and indicate if changes were made. The images or other third party material in this article are included in the article's Creative Commons license, unless indicated otherwise in a credit line to the material. If material is not included in the article's Creative Commons license and your intended use is not permitted by statutory regulation or exceeds the permitted use, you will need to obtain permission directly from the copyright holder. To view a copy of this license, visit http://creativecommons.org/ licenses/by/4.0/.

(c) The Author(s) 2019 